\title{
Flexible recognition of rapidly evolving promoter sequences by mitochondrial transcription factor 1
}

\author{
Robert P. Fisher, Melissa A. Parisi, and David A. Clayton \\ Department of Developmental Biology, Stanford University School of Medicine, Stanford, California 94305-5427 USA
}

\begin{abstract}
Transcriptional promoters of mitochondrial DNA have diverged extensively in the course of mammalian evolution. Nevertheless, the transcriptional machinery and the overall mechanisms of transcriptional control and regulation seem to be conserved. We have compared the human and murine homologs of the major DNAbinding transcriptional activator, mitochondrial transcription factor 1 (mtTF1), with unexpected results. Both proteins have similar chromatographic and transcriptional properties and are the same size. Both recognize and bind sequences between -12 and -39 within their respective homologous promoters. However, the sequences that they recognize are markedly divergent; although the base pairs they contact are situated similarly or identically with respect to the transcriptional start site, sequence identity between the two species' contact points is $<\mathbf{5 0 \%}$. Interestingly, the two proteins are functionally interchangeable; each can bind to the heterologous light-strand promoter and can activate transcription by the heterologous mitochondrial RNA polymerase. Thus, the RNA polymerase or some as yet undetected transcription factor, rather than mtTF1, may determine the strict species specificity of mitochondrial transcription. Flexible DNA sequence recognition by mtTF1, on the other hand, may be a principal facilitating mechanism for rapid control sequence evolution.
\end{abstract}

[Key Words: DNA-binding protein; evolution; mitochondria; promoter specificity; trans-acting factor; transcription activator]

Received August 21, 1989; revised version accepted October 16, 1989.

The transcriptional promoters of vertebrate mitochondrial DNA (mtDNA) have diverged rapidly, in contrast to the strict conservation of overall genomic structure, transcriptional organization, and regulation (for review, see Clayton 1984). Recent works have helped to define this paradoxical situation, through the identification and mapping of transcriptional control elements in mtDNA of several species and the concurrent dissection and characterization of the transcriptional machinery that recognizes these elements in homologous in vitro systems. In human mtDNA, both major transcriptional promoters [heavy $(\mathrm{H})$ - and light $(\mathrm{L})$-strand promoters; HSP and LSP] contain an upstream regulatory element necessary, but not sufficient, for efficient transcription; and at least one additional domain encompassing the transcription start site, which is essential for accurate initiation (Fisher et al. 1987; Topper and Clayton 1989). In mouse mtDNA, a similar picture emerged from in vitro deletion and linker-insertion mutational analyses of the LSP; the wild-type functional promoter consists of multiple discrete domains (Chang and Clayton 1986b).

Comparison of these two mammalian systems reveals similarities in the spatial organization and functional anatomy of mitochondrial promoters and, perhaps, in the differential regulation of the distinct transcription units encoded on the two strands of mtDNA; in both human and mouse in vitro systems, the LSP is a much stronger promoter than the HSP under most conditions tested (Chang and Clayton 1986a,b,c; Fisher and Clayton 1988). It is also clear from these studies that the similarity does not extend to the DNA sequence, where no homology between human and mouse promoters is apparent. However, these analyses, which have all focused on single isolated species, have so far shed little light on the unique evolutionary lability of mtDNA promoter sequences. Only by direct comparisons of functionally homologous factors and DNA sequence elements in divergent systems could we identify the determinants of species specificity and begin to understand the mechanisms driving (or allowing) promoter divergence.

Mitochondrial transcription factor 1 (mtTF1) is a 25kilodalton $(\mathrm{kD})$ protein that has been purified to homogeneity from human cells (Fisher and Clayton 1988) and that activates transcription by binding specifically to the upstream control regions of the human HSP and LSP (Fisher et al. 1987). The human mitochondrial RNA (mtRNA) polymerase has been partially purified but has not been shown to bind specifically to mitochondrial promoters, either in the presence or absence of mtTFl binding (R.P. Fisher and D.A. Clayton, unpubl.).

Here we report the first analogous fractionation of a mouse mitochondrial transcription extract. The murine 
mtTF1 can be separated from mtRNA polymerase by chromatography on phosphocellulose, is required for efficient in vitro transcription initiation at the LSP by isolated polymerase, and binds specifically and tightly to an upstream regulatory element of the LSP located between 11 and $39 \mathrm{bp}$ upstream of the transcriptional start site, a region with no evident sequence homology to its human counterpart. Methylation interference analysis of protein-DNA contacts made by human and mouse mtTF1 and their respective LSPs demonstrates interactions with bases in similar, or even identical, positions relative to the transcriptional start site but with little homology between the individual base pairs contacted.

Attempts to 'reprogram' mitochondrial transcription by inducing either human or mouse mtRNA polymerase to transcribe the heterologous LSP in the presence of mtTF1 specific for the template DNA proved unsuccessful. However, mtTFl purified from mouse mitochondria could stimulate transcription of the human LSP by factor-depleted human polymerase. Likewise, human mtTF1 could stimulate mouse mtRNA polymerase to recognize the mouse LSP. Furthermore, DNase I footprint analysis demonstrated specific binding of each factor to the heterologous LSP, albeit with reduced affinity. These results suggest a remarkable flexibility in promoter recognition by mtTF1 and may reflect an adaptation to, or a precondition for, rapid promoter divergence.

\section{Results}

\section{Purification of mtTF1 from mouse mitochondria}

We sought to identify and isolate the mouse analog of human mtTFl by adapting the purification scheme and specific assays developed for the human factor (Fisher and Clayton 1988). An eluate from a DEAE column, designated the DEAE pool, supports transcription from the LSP of mouse mtDNA (see Fig. 9). DNase I footprint analysis, using the DEAE pool and a ${ }^{32}$ P-labeled fragment of cloned mouse mtDNA containing an intact LSP, revealed the presence of an activity that binds upstream of the transcriptional start site, protecting a region of $\sim 25$ bp. Moreover, this fraction had DNAbinding activity in a gel retardation assay with the same labeled fragment. We were encouraged to believe that this activity was indeed the mtTF1 equivalent, both by similarities in the DNase I footprint (see below) and by its virtually identical behavior in the gel retardation assay; using DNA fragments of similar length, human mtTFl and the putative mouse mtTFl produced electrophoretic mobility shifts of the same magnitude, with the same ladder-like distribution of fragments that, in the case of human mtTF1, has been shown to reflect complexes of varying stoichiometry (data not shown). Furthermore, mouse $\mathrm{mtTF} 1$, isolated as an $\sim 25-\mathrm{kD}$ species from a denaturing gel can, upon renaturation, recognize the mouse LSP by a gel retardation assay (data not shown); this is true for human mtTF1 as well (Fisher and Clayton 1988).
In ion-exchange chromatography on phosphocellulose, the DNA-binding activity, which we followed using the gel retardation assay, elutes with an ionic strength of $\sim 0.7 \mathrm{M} \mathrm{KCl}$, as does human mtTF1 when chromatographed on the same resin (Fisher and Clayton 1985). Like the human factor, the mouse activity is required for reconstitution of specific transcription (see Fig. 9 below). In the DNase I protection experiment shown in Figure 1, increasing amounts of this transcription-stimulatory activity were incubated with ${ }^{32} \mathrm{P}$-endlabeled LSP-containing DNA fragment prior to digestion with DNase I. A footprint is seen between positions -11 and -35 (relative to transcriptional start site +1 ) on the noncoding strand (Fig. 1A) and between -14 and -39 on the coding strand (Fig. 1B). This corresponds closely with a promoter domain of the mouse LSP, shown previously to be necessary for efficient promoter selection (Chang and Clayton 1986b). This location is virtually identical to that of the mtTF1-binding site in the human LSP (Fisher et al. 1987). The human and mouse LSPs (noncoding strand only) are aligned at their respective start sites in Figure 1C, and the limits of the footprinted region are indicated by shaded boxes over (mouse) and under (human) each sequence. The lack of homology suggests frequent mutation of the DNA target sequence, even in the face of spatial constraints on these control elements.

\section{Contacts between mtTF1 and the LSP in human and mouse mtDNA}

We have mapped the contacts between mtTF1 and specific base pairs of the LSP that stabilize the proteinDNA complex, both for human and mouse mitochondria, using a methylation interference strategy (Siebenlist and Gilbert 1980; Hendrickson and Schleif 1985). Partial methylation of LSP-containing DNA fragments, at N7 of guanine residues and at N3 of adenine residues by dimethylsulfate (DMS), should render a subset of the fragments - those methylated at bases contacted by mtTF1 - incapable of binding mtTF1 specifically (i.e., stably). Such experiments revealed that both human (Fig. 2) and mouse (Fig. 3) factors contact purine residues on both strands of the DNA in both the major and minor grooves in a region completely contained within the sequences that they protect from DNase I cleavage.

In Figure 4, human and mouse LSPs have been aligned at their transcriptional start sites, and the bases contacted by mtTFl (on either strand) have been circled. Comparison of the contacts made by human and mouse mtTFl at their homologous LSPs reveals a similar distribution. Both factors contact a proximal cluster of six residues between 17 and $22 \mathrm{bp}$ upstream of the start site and a more distal cluster between -31 and -35 of the human LSP or between -30 and -37 in mouse. Within these clusters, minimal sequence homology between the two species is apparent. Specifically, both human and mouse mtTF1 contact the sequence $5^{\prime}$-TPuAC- $3^{\prime}$ at the downstream (start-site proximal) boundaries of their respective binding sites, and the sequence $5^{\prime}-\mathrm{ACT}-3^{\prime}$ at 
Fisher et al.
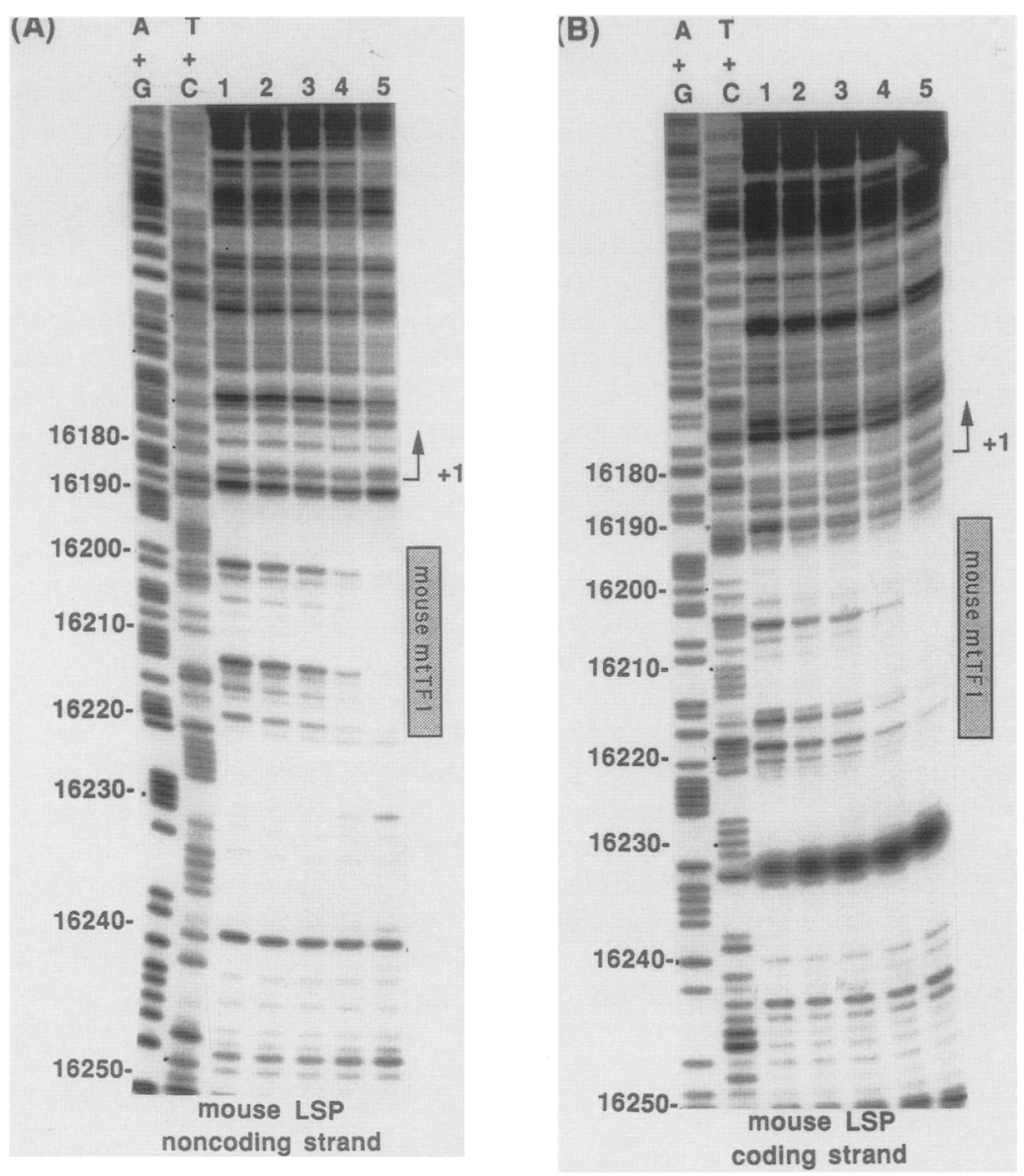

(C)

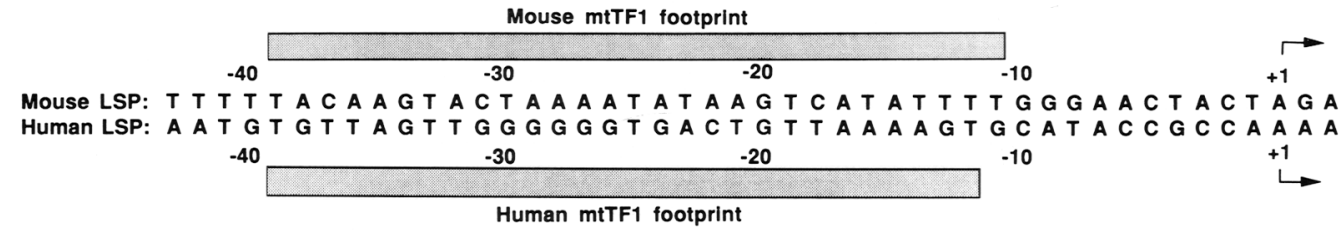

Figure 1. Identification of the mouse LSP mtTFl-binding site. The mouse mtDNA insert in deletion clone $\Delta 5^{\prime}-88$ (Chang and Clayton 1986b) was end-labeled with ${ }^{32} \mathrm{P}$ upstream of the transcriptional start site (nucleotide 16184) on either the noncoding $(A)$ or coding strand $(B)$. The labeled fragments were then incubated with increasing amounts of phosphocellulose-purified mouse mtTF1. The protein-DNA mixtures were subjected to limited digestion with DNase I and then deproteinized; the DNA was denatured and electrophoresed in $6 \%$ polyacrylamide, $7 \mathrm{M}$ urea gels, as described in Materials and methods. The amounts of factor added were none (lanes 1), $0.5 \mu \mathrm{l}$ (lanes 2), $1 \mu \mathrm{l}$ (lanes 3), $2 \mu \mathrm{l}$ (lanes 4), and $4 \mu \mathrm{l}$ (lanes 5). The same fragments were subjected to sequence-specific chemical cleavage (Maxam and Gilbert 1977) at purine (lanes $A+G$ ) or pyrimidine (lanes $T+C$ ) residues, and the denatured products were electrophoresed alongside the DNase I digestion products as markers. The numbers alongside the sequence ladders in this and subsequent figures derive from the mouse mtDNA sequence of Bibb et al. (1981). The region protected from DNase I digestion by $\mathrm{mtTFl}$ is indicated by the shaded bar $($ right $)$; this is an approximation due to the stretches of infrequently cleaved phosphodiester bonds found at both boundaries of the binding site. The arrow indicates the location of transcription initiation $(+1)$ and the direction of elongation. (C) The mtTF1-binding sites of mouse and human LSPs are compared. The two DNA sequences are aligned at their transcriptional start sites (position +1 , indicated by arrow), and the sequences protected by the homologous mtTFl are denoted by the shaded boxes over (mouse) or under (human) each sequence. Only the noncoding strand sequences are shown, but the footprinted region defined by the boxes includes all sequences protected on either strand; in both human and mouse LSPs, there is a three- or four-base staggering of the protected region between the two strands of DNA.

the upstream (start-site distal) boundaries. In addition, both LSPs appear to contain a repeated three- or fourbase sequence element that is critical for factor binding. Both domains contacted by mouse mtTF1 contain the sequence $5^{\prime}$-ACTT- 3 ', whereas human mtTF1 contacts two $5^{\prime}$-PyAAC-3' motifs. These repeats are equidistant from each other in both promoters ( $13 \mathrm{bp}$ between the first positions of each) but are shifted $2 \mathrm{bp}$ closer to the 

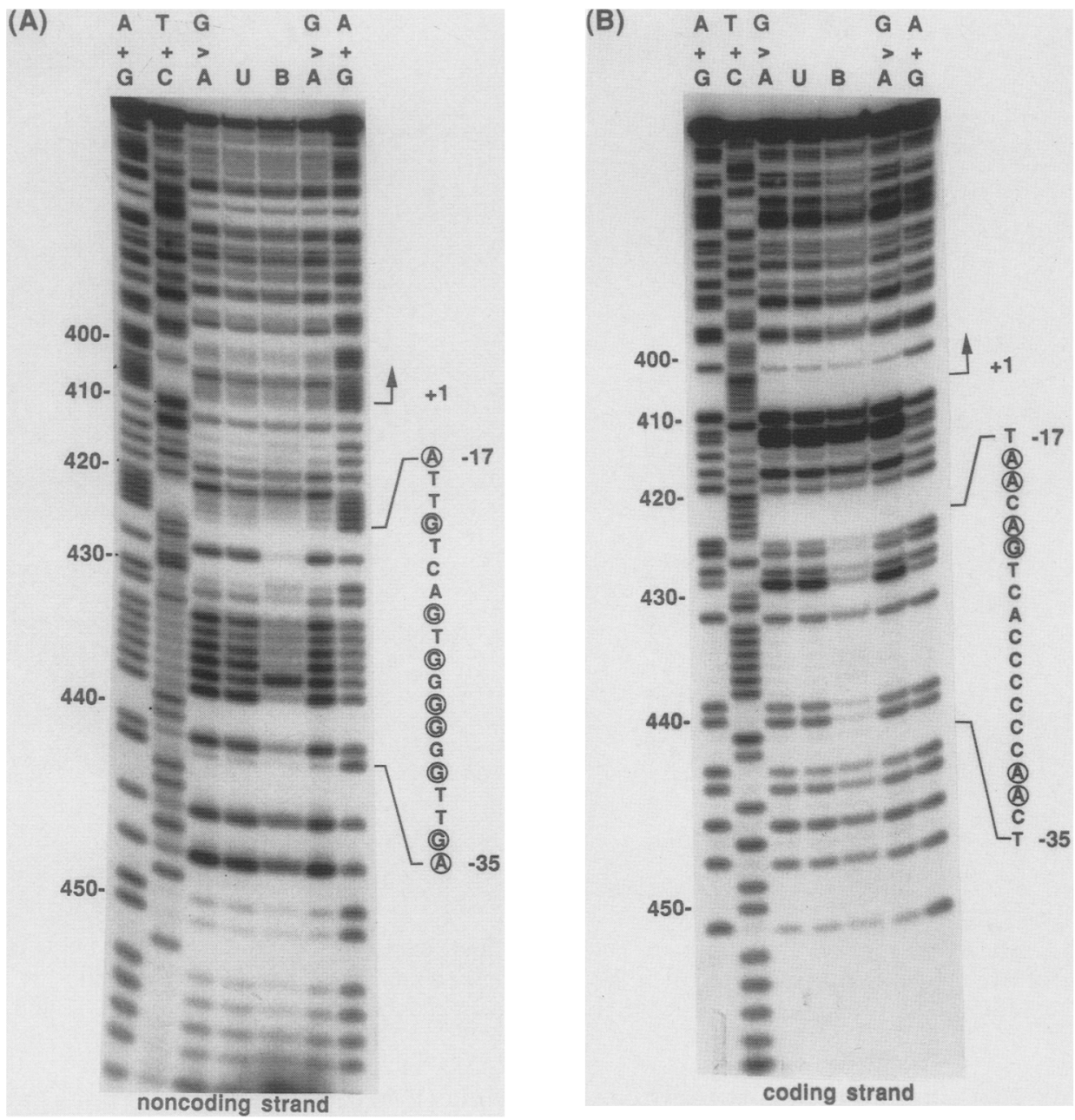

Figure 2. Contacts between human mtTFl and the human LSP identified by methylation interference. An LSP-containing fragment from human deletion clone $\mathrm{L}^{\prime} \Delta-56$ (Chang and Clayton 1984) was end-labeled with ${ }^{32} \mathrm{P}$ on either the noncoding $(A)$ or coding $\langle B|$ strand and treated with DMS for methylation interference analysis of mtTFl-DNA binding. The mixture of methylated labeled fragments was incubated with $4 \mu \mathrm{l}$ of phosphocellulose-purified mtTFl; nonspecific mtTF1-DNA complexes were dissociated by a 2-min incubation in the presence of excess unlabeled LSP-containing DNA, and the specific mtTF1-LSP complexes were separated from unbound (and dissociated) fragments by electrophoresis in nondenaturing gels. DNA was recovered from the fractionated gel and cleaved with piperidine at methylated guanine and adenine residues $(\mathrm{G}>\mathrm{A})$, denatured, and electrophoresed in sequencing gels. Cleavage products from the fraction of DNA fragments bound in specific complexes with mtTF1 were electrophoresed in lanes B, whereas fragments produced by cleavage of unbound DNA were electrophoresed in lanes U. The same fragments were subjected to Maxam-Gilbert (1977) chemical cleavage reactions, without exposure to mtTF1 or electrophoresis in native gels, to produce the sequence ladders seen in lanes $A+G, T+C$, and $G>A$. Human mtDNA sequences in this and subsequent figures are numbered according to Anderson et al. (1981), and the start site $(+1$ is nucleotide 407$)$ and direction of transcription are indicated by the arrow at right in $A$ and $B$. The sequences of the contacted region (between the first and last contacted bases) are also shown at right, with each contacted base circled.

transcriptional start site in the human LSP. A third repeat of $5^{\prime}$-PyAAC-3', immediately upstream of the second copy in the human LSP (see Fig. 4) does not appear to be contacted, or is only weakly contacted /see Fig. 2). This may reflect a requirement for correct spacing between the repeated elements for the two domains to cooperate in the stable binding of an mtTFl molecule.

Protein-DNA contacts that are not absolutely essential for stable binding or, alternatively, not completely disrupted by methylation, are a prominent feature of the human mtTFl-LSP interaction. These contacts are represented by species that are diminished in, but not completely absent from, the bound fraction (lanes B in Fig. 2) compared with the unbound fraction (lanes U). They lie primarily between the two domains described above and may help fix the protein in the proper position to activate transcription, perhaps by preventing 'slippage' of $\mathrm{mtTF} 1$ to the third upstream repeat of the $5^{\prime}$-PyAAC-3' element (see above). The importance of this cluster of $\mathrm{G}$ residues on the noncoding strand is suggested by phylogenetic comparisons of primate LSP sequences /see Fig. 
Fisher et al.
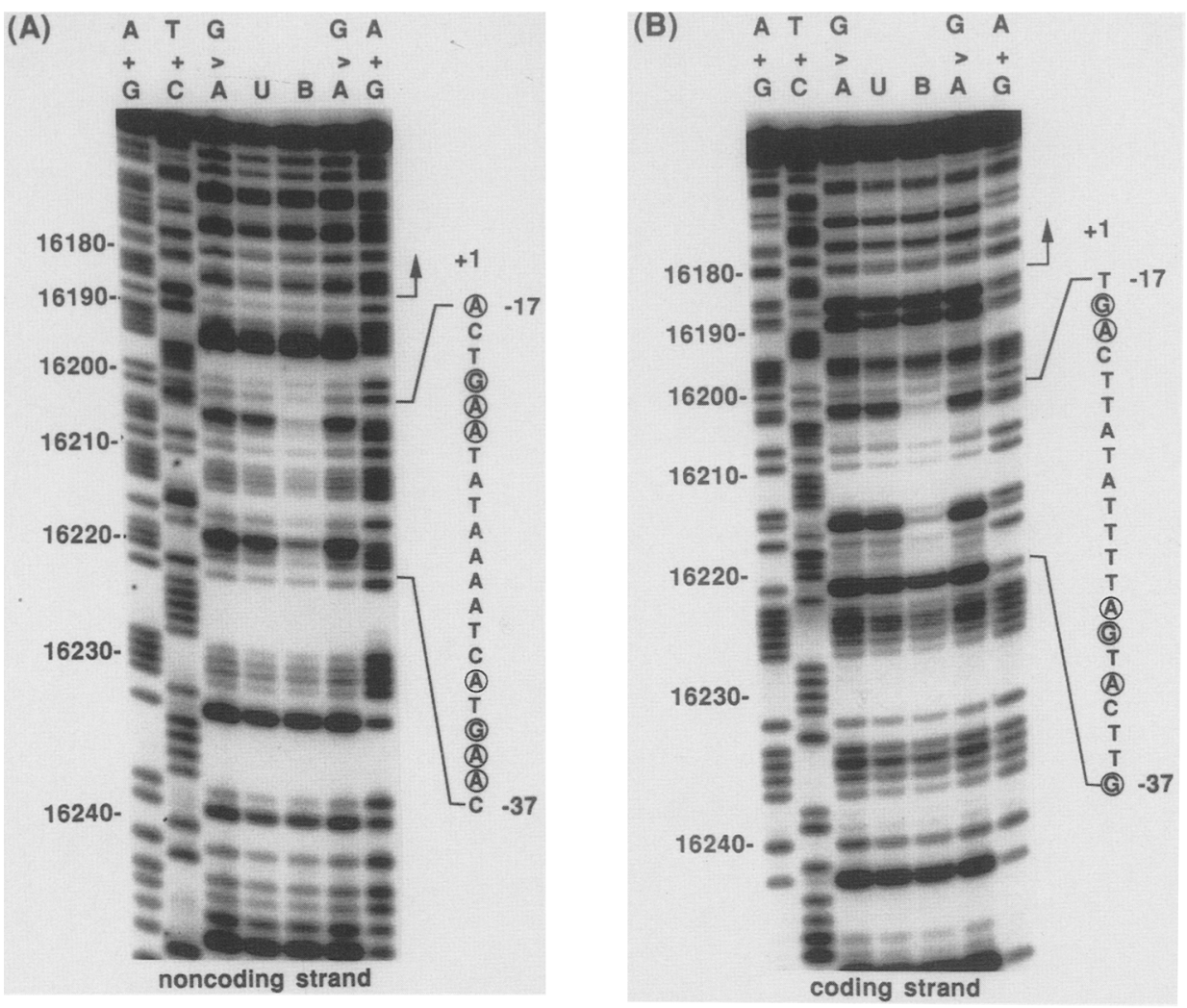

Figure 3. Contacts between mouse mtTF1 and the mouse LSP identified by methylation interference. This is the same as Fig. 2, except that the labeled fragment was derived from mouse deletion clone $\Delta 5^{\prime}-88$ (Chang and Clayton $1986 \mathrm{~b}$ ), and we used $4 \mu \mathrm{l}$ of a $\mathrm{mtTF} l$ fraction that was purified from a mouse mitochondrial extract by sequential chromatography on DEAE and phosphocellulose columns.

10 and Discussion). This cluster has been strongly conserved among the ape LSPs (Foran et al. 1988); however, in the mouse LSP, the sequence between the two contacted regions is very AT-rich, and none of the A residues appears to be contacted by mtTFl at N3 (Figs. 3 and 4). Caution must be exercised, however, in the interpretation of the mouse methylation interference pattern at $\mathrm{A}$ residues, as even the largest diminutions (indicating contact points) are subtle. Where possible, we have used comparisons between a suspected contact point and a neighboring uncontacted $\mathrm{A}$ residue to identify critical residues. A more conservative approach, considering only $\mathrm{G}$ residues, might well be justified.

On the basis of the above methylation-interference data and assuming a B-DNA configuration, we have generated three-dimensional models of both human and mouse mtTFl-LSP complexes. Several views of each complex are shown in Figure 5 to illustrate similarities and differences between the two species. Views A and C, which show only major groove contacts (green hemispheres delimit van der Waals radii at the N7 positions of contacted guanine residues), seem to suggest a marked difference between the modes of recognition in the two species. Human mtTF1 makes extensive contacts in the major groove of the DNA along the entire length of the binding site; one can envision a surface of the protein in close apposition to the major groove for two helical turns. At first glance, the mouse contact pattern is much simpler, consisting of two clusters of major groove contacts at opposite ends of the binding site, separated by one helical turn that is not contacted by protein. However, it must be remembered that between these two 'domains' of the murine mtTFl-binding site there are no G-C base pairs, which are prerequisite for mapping major groove contacts by methylation interference. Thus, murine mtTFl could very well lie along the major groove in a extended protein-DNA complex, similar to its human counterpart.

In views $B$ and $D$, minor groove contacts have been added to the model (red hemispheres), and the molecules have been rotated about the long axis $\sim 180^{\circ}$. Mouse mtTF1 does not use minor groove contacts in the internal AT-rich region of its recognition sequence to stabilize binding - a fact that may argue in favor of undetectable major groove contacts in this region resembling those made by human mtTF1. Where it was possible to map major and minor groove contacts in the mouse complex, considerable similarity between the two species is evident. Conservation of contact positions suggests that both human and mouse factors may approach the helical grooves from similar angles; both factors make extensive contact with both major and minor grooves in approximately the same locations relative to the start site. As predicted by the sequence ho- 


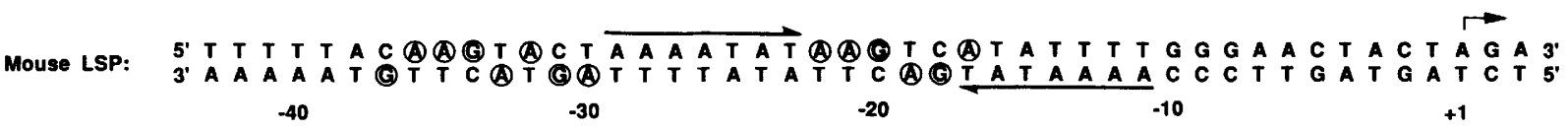

Figure 4. The structure of mammalian mtTF1-LSP complexes. The DNA sequences (noncoding strand at top) of both human and mouse mitochondrial LSPs have been aligned, using their respective transcriptional start sites (indicated by arrows above sequence) as reference points (labeled +1 ). Bases contacted by the homologous mtTF1, as determined by methylation interference (see Figs. 2 and 3 above) are circled. The mouse LSP contains inverted repeats of the sequence 5'-AAAATAT-3' (Chang and Clayton 1986b), as indicated by the longer arrows delimiting these elements.

mologies, some contacts are identical, some have apparently switched strands, and others seem to have been replaced by contacts at neighboring or nearby positions. Aside from the uncontacted (or perhaps 'silently' contacted) internal region of the mouse LSP, the largest discrepancy between the two models is the extension of mouse mtTF1-LSP contacts two bases farther upstream from the start site. It should be noted, however, that in some experiments, the human mtTFl appears to contact bases 2-4 nucleotides farther upstream than the 5 '-most contact shown (see Fig. 2). We have not established these as bona fide contacts, largely because of intractable artifacts specifically affecting very small fragments in our gels.

The molecules were rotated to sight down the long axis of the helix, both in the direction of transcription, i.e., toward the start site (Fig. 5E,G), and in the opposite direction, away from the start site (Fig. 5F,H). These projections show that both human and mouse factors make contacts on more than one face of the double helix and that mtTFl may wrap around the DNA in extended conformation. Similarities between human and mouse complexes are apparent in both views, consistent with the notion that the sequence specificity of the two proteins may have diverged without gross structural changes in the DNA-binding domain(s).

\section{Mouse mtTF1 binds to the human LSP in vitro}

It was known that human mitochondrial extracts could not function with mouse mtDNA promoters, and vice versa (see Figs. 7 and 9 below; Chang et al. 1985). We therefore attempted a series of heterologous DNA binding experiments to determine whether mouse
mtTF1 could bind specifically to the heterologous (human) LSP. Preincubation of a DNA fragment containing the human LSP with human mtTFl (Fig. 6, lane 5) results in DNase I protection of the mtTFl-binding site between -12 and -35 on the noncoding strand (A) and between -16 and -39 on the coding strand (B). Mouse mtTFl protects the same sequences in the human LSP but with a lower affinity, as judged by the reduced clarity of the resulting DNase I footprint; at the concentration of mouse mtTF1 required for significant protection of the human mtTFl-binding site, nonspecific binding to nonpromoter DNA sequences is seen (Fig. 6A,B, lanes 2-4). In addition, there are qualitative differences between the homologous and heterologous interactions discernible by footprint analysis. In binding to its target sequence within the human LSP, human mtTFl causes enhancement of DNase I cleavage at sites immediately adjacent to the protected region, at positions -10 and -38 on the noncoding strand, and between -6 and -14 and at position -41 on the coding strand. These cleavage enhancements are not seen when mouse mtTF1 binds to the human LSP; instead, enhanced cleavage sites appear on the noncoding strand at -2 , downstream of the protected region, and at -46 on the noncoding strand and at -48 on the coding strand, upstream of the footprint. None of these bonds is affected by binding of the homologous transcription factor; however, all three sites are located $7-8 \mathrm{bp}$ away from a position of cleavage enhancement in the homologous' footprint. Thus, the displacement may stem from geometric differences between the human and mouse factor molecules or between the structures of homologous and heterologous protein-DNA complexes and not directly from a difference in the sequence specificity of the two factors.

Figure 5. (See following page for figure.) Three-dimensional models of both human and mouse mtTF1-LSP complexes derived from methylation interference data. Corresponding stretches of mouse and human mtDNA $(-10$ to -40$)$ were constructed in the B-DNA configuration (chosen arbitrarily). To situate the proteins on the DNA molecules, the van der Waals radius of each atom at which methylation interferes with binding was labeled with a colored hemisphere (green hemispheres: N7 of guanine; red: N3 of adenine). In addition, the dyad symmetry within the murine LSP region has been indicated by coloring all residues gray within both repeats. Views A, B, E, and F are of the human mtTFl-LSP complex; views C, D, G, and H are of the mouse complex. In views A and C, only major groove contacts are highlighted (green hemispheres); in views $\mathrm{B}$ and $\mathrm{D}$, both major (green) and minor (red) groove contacts are indicated. In longitudinal views, the direction of transcription is rightward (although the start site is not actually shown), and for each pair (A and C, B and D), both human and mouse DNA molecules are shown in the same orientation (the same rotation about the long axis). Views $\mathrm{E}$ and $\mathrm{G}$ look down the long axis of the DNA in the direction of the start site and thus accentuate contacts at the upstream end of the binding site. Views $\mathrm{F}$ and $\mathrm{H}$ look away from the start site, highlighting the proximal cluster of contacts. 
Fisher et al.
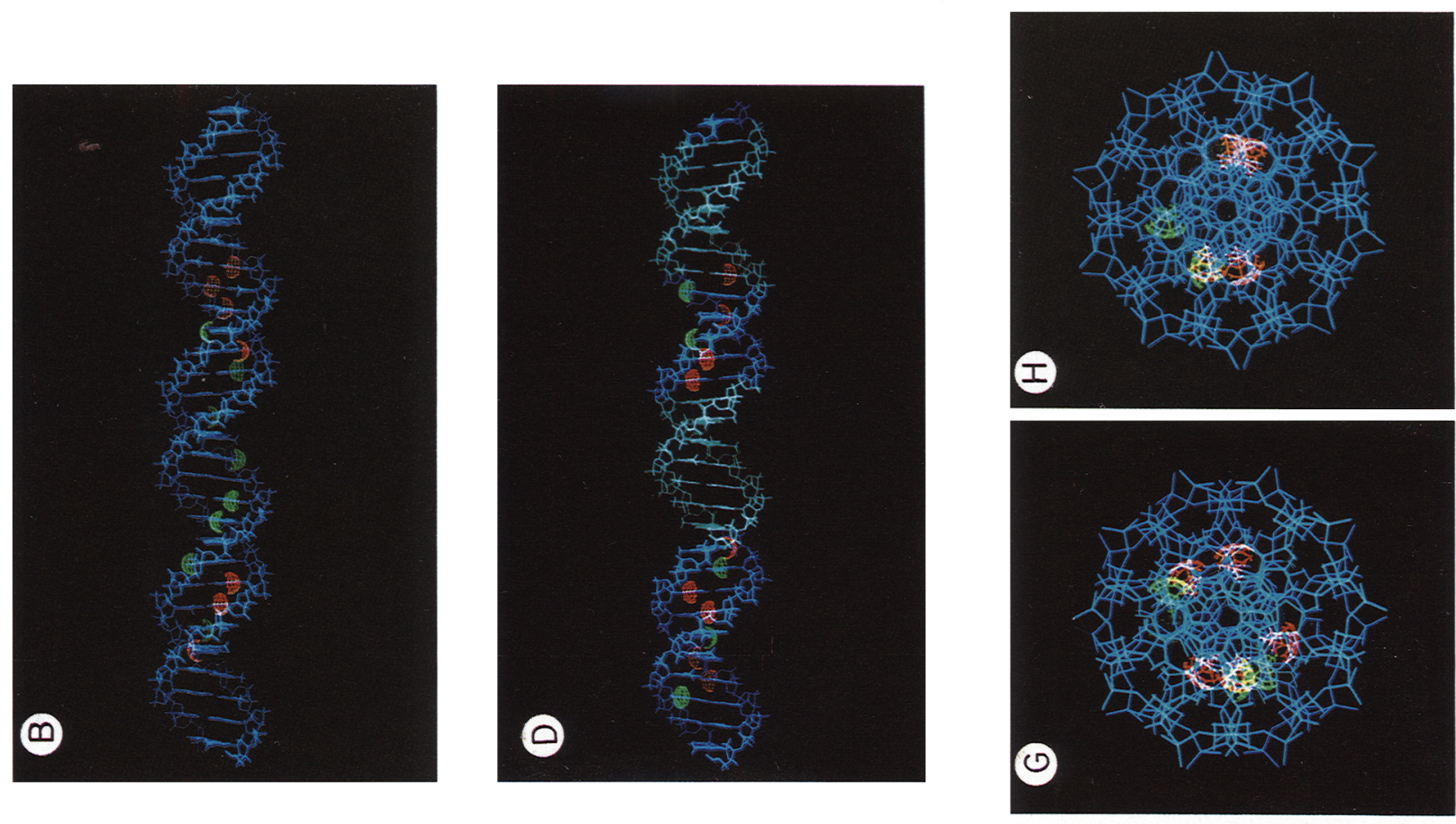

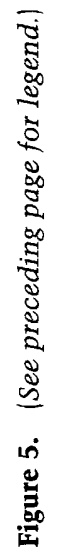
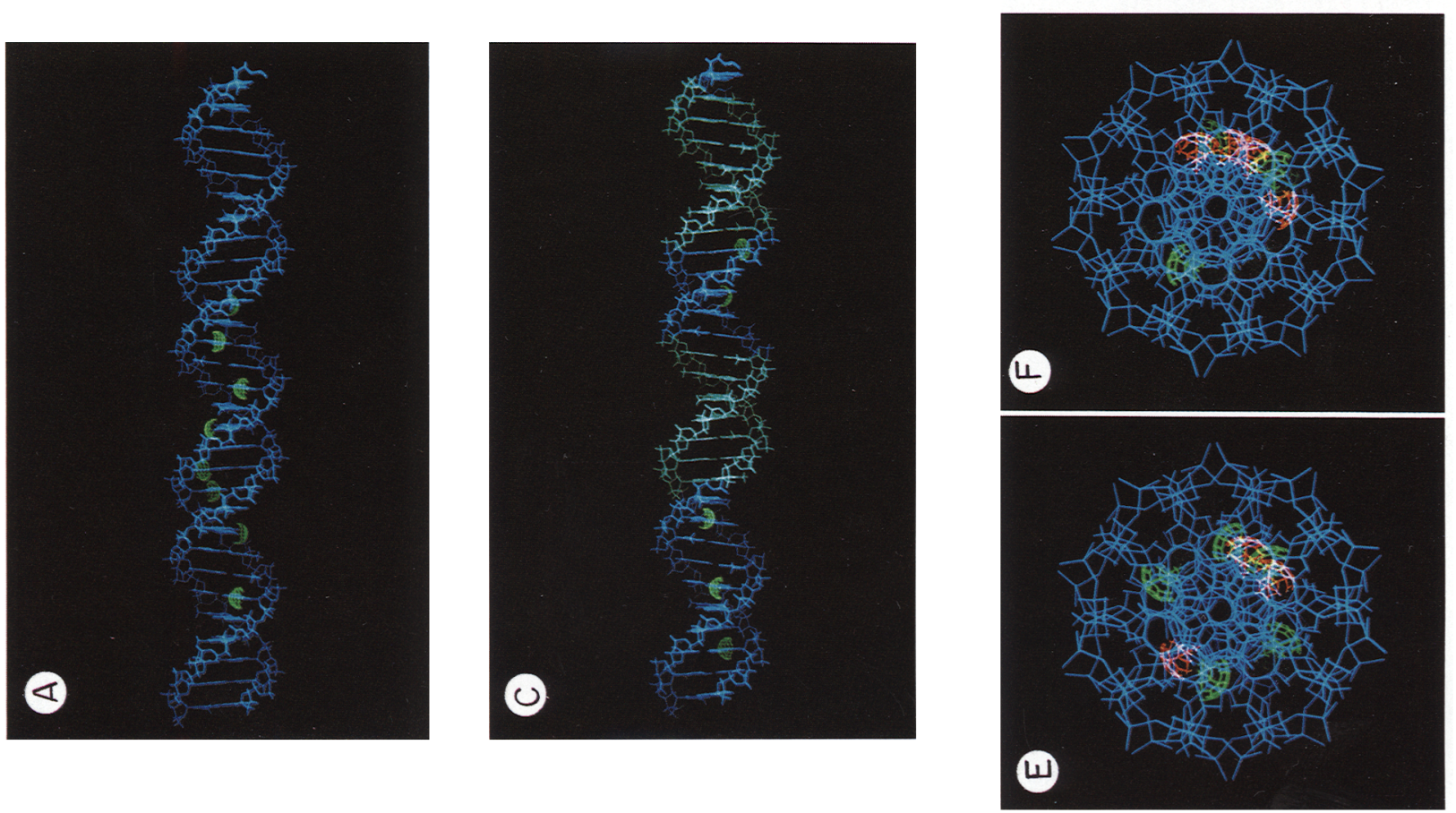

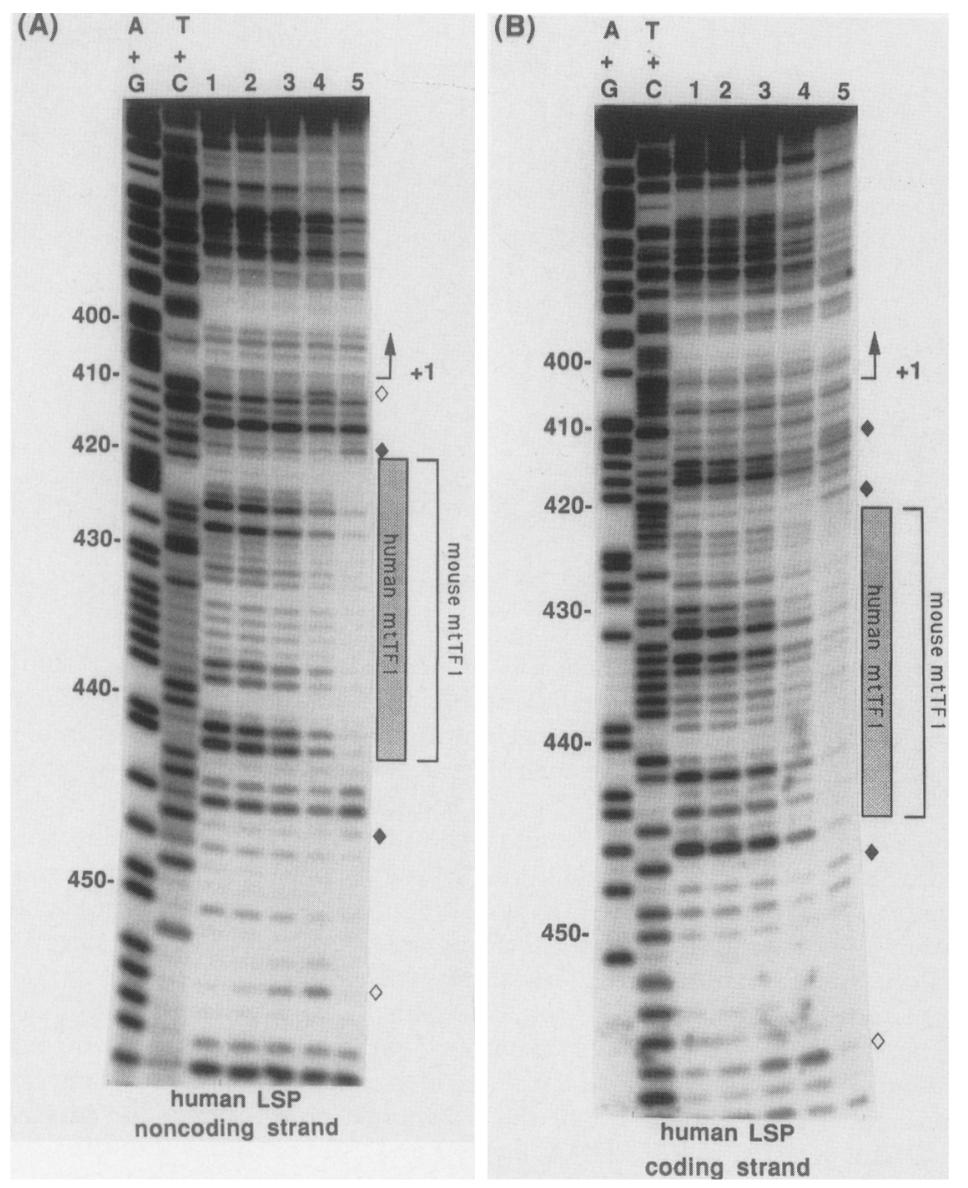

Figure 6. Heterologous footprinting of the human LSP by mouse mtTF1. The human LSP-containing fragment (see Fig. 2) was incubated with increasing amounts of the phosphocellulose-purified mouse mtTFl prior to partial DNase I digestion. $(A)$ The DNA is end-labeled with ${ }^{32} \mathrm{P}$ on the noncoding strand; $(B)$ the coding strand is end-labeled with ${ }^{32} \mathrm{P}$. The fragment was incubated with no added factor in lanes 1 and with $2 \mu \mathrm{l}$ (lanes 2), 4 $\mu 1$ (lanes 3), and $8 \mu l$ (lanes 4) of mouse mtTF1. In lanes 5 , the fragment was incubated with $4 \mu l$ of phosphocellulose-purified human mtTFl to generate the control homologous protection and enhancement pattern. Lanes $\mathrm{A}+\mathrm{G}$ and $\mathrm{T}+\mathrm{C}$ contain the products of Maxam-Gilbert sequencing reactions performed with the same fragment. Arrows at right denote the +1 position, where transcription initiates (nucleotide 407), and the direction of transcription elongation. The shaded bar delimits the sequence protected by human mtTFl (lanes 5); the bracket indicates the location (identical) of the heterologous footprint produced by mouse mtTF1 (lanes 2-4). Diamonds denote positions of cleavage enhancement (see text); $(\downarrow)$ bonds rendered hypersensitive by the homologous (human) factor; $|\diamond|$ sites where cleavage is enhanced by heterologous (mouse) factor binding.

Is the heterologous complex between murine mtTF1 and the human LSP transcriptionally competent?

Thus, mouse mtTF1 can recognize and bind specifically to the human LSP in virtually the same location as the homologous human factor. However, mouse mitochondrial transcription extracts alone are incapable of recognizing and initiating transcription at human mtDNA promoters, as demonstrated by the in vitro transcription experiment shown in Figure 7 . In lanes $12-15$, increasing amounts of a DEAE pool prepared from mouse mitochondria were incubated with a linear template DNA containing both the LSP and HSP of human mtDNA. This enzyme fraction is capable of accurate and efficient transcription initiation at mouse mtDNA promoters (see Fig. 9 below), yet it produces no correct transcripts when challenged with a human template. Dissociation, by phosphocellulose chromatography, of both human and mouse mtTFls from their respective homologous polymerases made it possible to ask whether this species specificity was the result of a nonfunctional mtTF1-LSP interaction, despite the ability of mouse mtTF1 to footprint the human LSP, or whether some other component of the transcriptional machinery posed the barrier to cross-species transcription. Attempts to reprogram the mouse mtRNA polymerase to recognize the human LSP, by supplementing the reac- tion with purified human mtTF1, were unsuccessful; no signal is produced above the background level generated by the human mtTF1 fraction (contaminated slightly with human polymerase) alone [Fig. 7; cf. lanes 3-6 (without mouse extract) with lanes 8-11 (with mouse extract)]. However, when human mtRNA polymerase depleted of human mtTF1 is supplemented with mouse mtTF1, transcription initiation at the human LSP is restored (Fig. 7, lanes 20-23). Thus, it appears that murine mtTF1 can substitute reasonably efficiently for its human homolog, consistent with the results of heterologous footprint analysis (see Fig. 6, above). This result also implies that species specificity resides in the fraction containing mtRNA polymerase-possibly in the polymerase itself.

\section{Human mtTF1 can bind and activate the murine LSP}

Very similar results were obtained when human mtTF1 was substituted for mouse mtTF1 in in vitro DNA binding and transcription assays with template fragments containing the mouse LSP sequence. In Figure 8, the results of a DNase I footprint analysis are shown; phosphocellulose-purified human mtTF1 (Fig. 8A,B, lanes 2-4) protects the same sequences upstream of the transcriptional start site as does the mouse factor (lanes 6). As with the mouse mtTF1-human LSP interaction, 


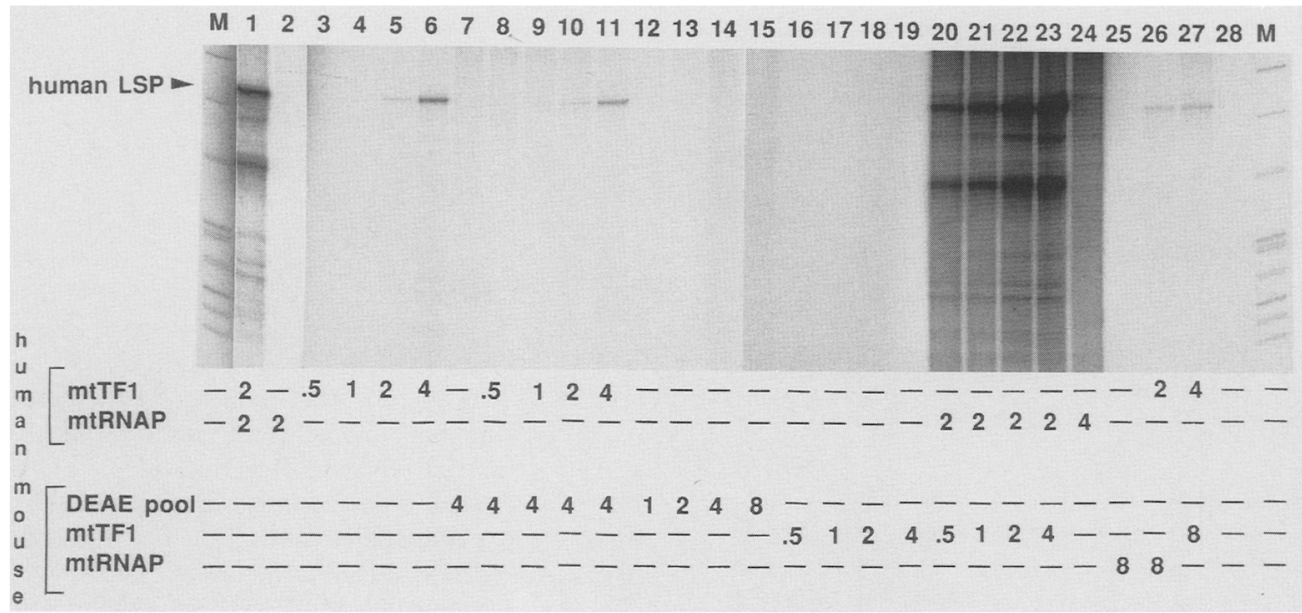

Figure 7. Heterologous in vitro transcription of the human mtDNA LSP. The human LSP, contained on a fragment of plasmid pKB741SP (Fisher and Clayton 1985), was used to program in vitro runoff transcription by mixtures of human and mouse mitochondrial protein fractions. The fractions and amounts (in microliters) added to each reaction are indicated below each lane. Human and mouse mtTF1 and mtRNAP refer to phosphocellulose-purified mtTF1 and mtRNA polymerase, respectively; the DEAE pool is a more complex fraction of mouse mitochondrial proteins comprising the bulk of both mtTFl and the polymerase isee Materials and methods). Transcription initiating at the correct LSP start site and elongating to the end of the template fragment produces an 416-nucleotide runoff transcript indicated by the arrowhead at left. Also visible are additional species representing paused, terminated, or processed transcripts that are routinely observed in these assays (see Fisher and Clayton 1988). The experimental design may be summarized as follows: (Lane 1) Homologous transcription by human mtTFl and mtRNA polymerase; (lane 2) human mtRNA polymerase alone; (lanes 3-6) a titration of human mtTFl alone; (lane 7) mouse DEAE pool alone; (lanes 8-11) a titration of human mtTF1 in the presence of mouse DEAE pool; (lanes 12-15) a titration of mouse DEAE pool alone; (lanes 16-19) a titration of mouse mtTF1; (lanes 20-23) mouse mtTF1 titrated in the presence of $2 \mu \mathrm{l}$ human mtRNA polymerase (cf. with lane 2; human polymerase with no added factor); (lane 24) twice the amount $(4 \mu \mathrm{l})$ of human mtRNA polymerase with no added transcription factor; (lanes 25 and 26) mouse mtRNA polymerase without and with human mtTFl, respectively; (lane 27) a combination of human and murine mtTF1; (lane 28) template alone; (lanes $M$ ) a ${ }^{32}$ P-end-labeled Hpall digest of pBR322 DNA. Lanes 1 and 2 are of shorter exposure.

the affinity of human mtTF1 for the heterologous LSP is lower, and the pattern of protein-induced cleavage enhancement differs markedly between the homologous and heterologous footprints. In general, cleavage enhancement seems to be more pronounced when human mtTFl interacts with DNA; bonds at both boundaries of the mouse control element are rendered hypersensitive to hydrolysis by the binding of human, but not mouse, mtTF1. It thus seems unlikely that these hypersensitive sites are integral to the sequence specificity or transcriptional activity of these mitochondrial transcription factors.

More puzzling is the binding of human mtTFl for some contaminating human activity) to a sequence farther upstream of the mouse LSP transcriptional start site. This protection extends from position -64 to at least -80 and may involve the end of the fragment (up to -88 ). This does not represent nonspecific binding to fragment ends, as it does not occur with other fragments (see Fig. 6 above), nor does it correspond to any footprints induced by a homologous mouse activity; no protection of this region appears when this fragment is incubated with mouse mtTF1 (Fig. 8, lanes 6; Fig. 1) or with cruder mouse mitochondrial fractions (M.A. Parisi and D.A. Clayton, unpubl.). Within this sequence are several fragmentary similarities to the human mtTF1binding determinants; however, neither the linear sequence homology nor the spacing of these candidate contact points seems sufficient to explain the very high apparent affinity of the interaction. Proximity to fragment ends may contribute to the tight binding; alternatively, a contaminating DNA-binding activity may be responsible.

In Figure 9, the results of runoff transcriptions using both homologous and heterologous combinations of protein fractions with a mouse LSP sequence as template are shown. Like its human counterpart, mouse mtTFl is unable to alter the specificity of the heterologous (human) transcription machinery to recognize the mouse promoter. This was tested using both phosphocellulose-purified (factor-depleted) human mtRNA polymerase (lanes 7-10) and the unfractionated DEAE pool of human mitochondrial proteins (lane 24), which conceivably could contain additional factors necessary for optimal promoter recognition. In contrast, the human mtTF1 fraction can stimulate transcription from the mouse LSP catalyzed by mouse mtRNA polymerase that has been depleted of endogenous mouse mtTFl (Fig. 9, lanes 13-16).

That this heterologous transcription stimulation is due to the binding of human mtTF1 to the upstream control element of the mouse LSP seen in footprint analysis (Fig. 8) seems likely. However, two alternative explanations are consistent with the above data. The most obvious, that stimulation actually stems from the tight binding of human mtTF1 to a cryptic recognition site 

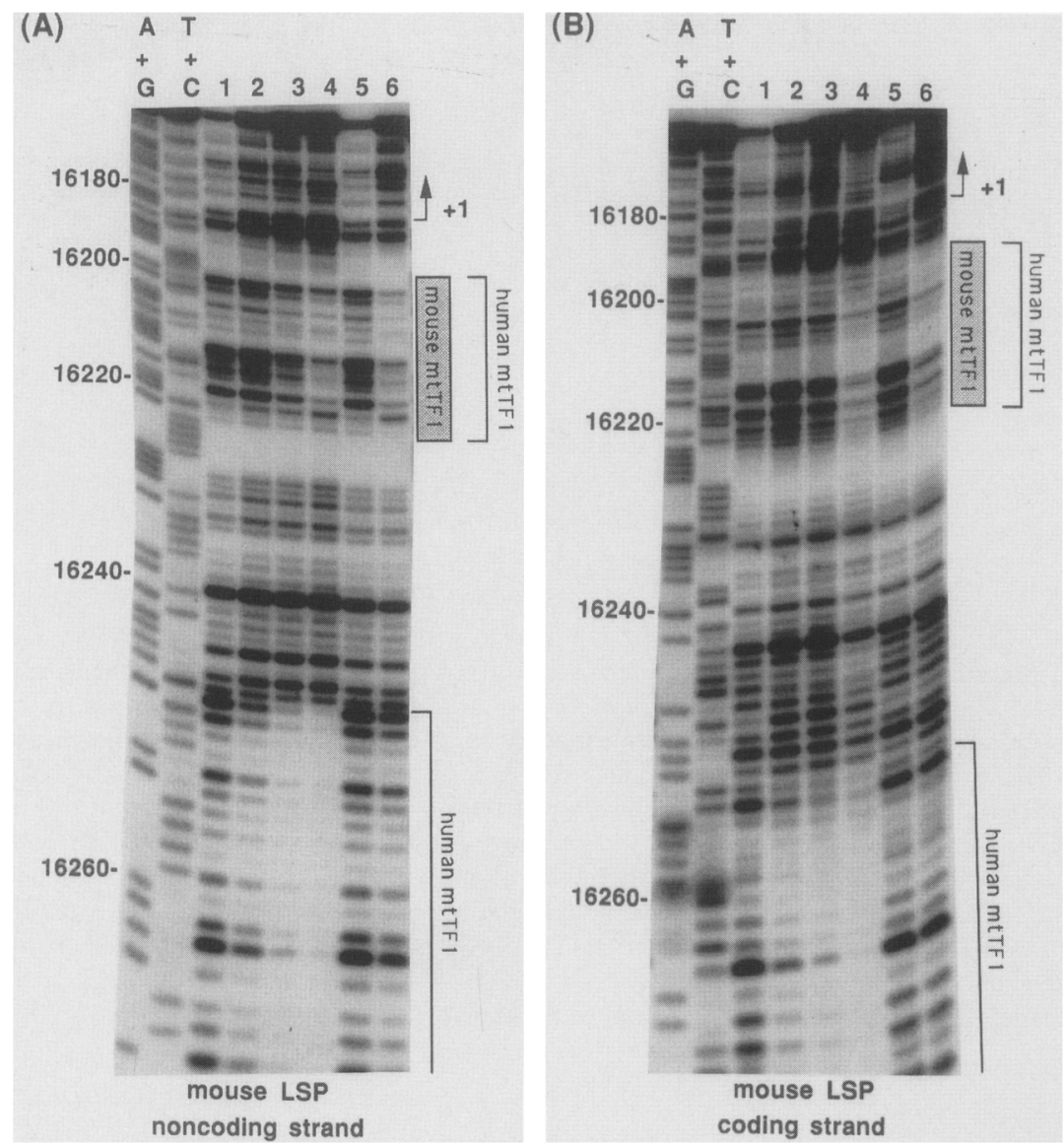

Figure 8. Heterologous footprinting of the mouse LSP by human mt'TF1. As in Fig. 1, the DNA fragment contains a mouse LSP labeled $\sim 88$ bp upstream of the transcriptional start site $(+1$ is nucleotide 16184$)$, on either the noncoding $(A)$ or coding $(B)$ strand. The fragment was incubated with either human (lanes 2-4) or mouse (lanes 6) phosphocellulose-purified mtTF1 prior to DNase I digestion. Factor additions were no factor added (lanes 1 and 5); $2 \mu l$ human mtTF1 (lanes 2); $4 \mu \mathrm{l}$ human mtTFl (lanes 3); $8 \mu l$ human mtTF1 (lanes 4); and $6 \mu \mathrm{l}$ mouse mtTF1 (lanes 6 ). The homologous mouse footprint is indicated by the shaded bar; brackets are used to delimit the sequences protected by the heterologous factor, human mtTF1, both in the mouse LSP and at a site farther upstream, where no homologous mouse protection is evident.

upstream of -64 (see Fig. 8 above), is excluded by the fact that this site can be deleted without an effect on the transcriptional response to human mtTFl /data not shown). A second caveat arises from the use of a partially purified human mtTFl preparation for the preceding experiments; heterologous stimulation may be a function of some as yet unidentified non-species-specific transcription factor distinct from mtTF1. This possibility was excluded by the use of a homogeneous preparation of human mtTF1 containing only a single major polypeptide species (Fisher and Clayton 1988). Homogeneous human mtTF1 stimulated heterologous transcription with the same efficiency as did partially purified mtTF1 (data not shown). Thus, we can conclude that human mtTF1 can bind to the murine LSP upstream element - a sequence only distantly related to its natural target-and interact with a heterologous mouse polymerase (and perhaps other factors) to stimulate accurate transcription from the heterologous LSP.

\section{Discussion}

\section{Rapid evolution of mitochondrial promoter sequences}

A comparison of the human LSP with the putative promoter regions, identified by homology with the human sequence and by location on the circular genome, of several ape mtDNAs (Foran et al. 1988; J.L. Boore and W.M. Brown, pers. comm.) is shown in Figure 10A. Also in- cluded is a putative bovine LSP region, identified by its proximity to the $5^{\prime}$ ends of in vivo L-strand transcripts (King and Low 1987). Base pairs contacted by human mtTFl in its homologous target sequence are denoted by circled bases in the human sequence (top line). For each species, only those positions that differ from the human sequence are specified on the appropriate line below. Remarkably, the start site-proximal sequence 5'TAACAG-3' (coding strand sequence), which comprises six of the strongest contacts detected by methylation interference (see Figs. 2 and 4), has been conserved in all of the ape mtDNAs sequenced, except for that of orangutan, where the first base has undergone a T-to- $\mathrm{C}$ transition. This hexameric sequence is also found in a putative bovine LSP, as noted previously (King and Low 1987). Human, gorilla, and pygmy chimpanzee mtDNAs differ at only a single position [position 437 in the human mtDNA sequence of Anderson et al. (1981)], which is a $\mathrm{C}$ in human, but a $\mathrm{T}$ in the other three species. Common chimpanzee also has a $\mathrm{T}$ at this position, and one additional C-to- $T$ transition four bases upstream. The latter change is notable, as it occurs at a base pair that contacts mtTF1. In contrast, the more common transition, $C$ to $T$ at position 437 , would not be expected to affect mtTF1 binding, based on methylation interference results (Fig. 2A). This $\mathrm{C}$ is complementary to the single noncontacted $G$ residue that appears in a cluster of contacted $\mathrm{G}$ residues on the noncoding strand. 


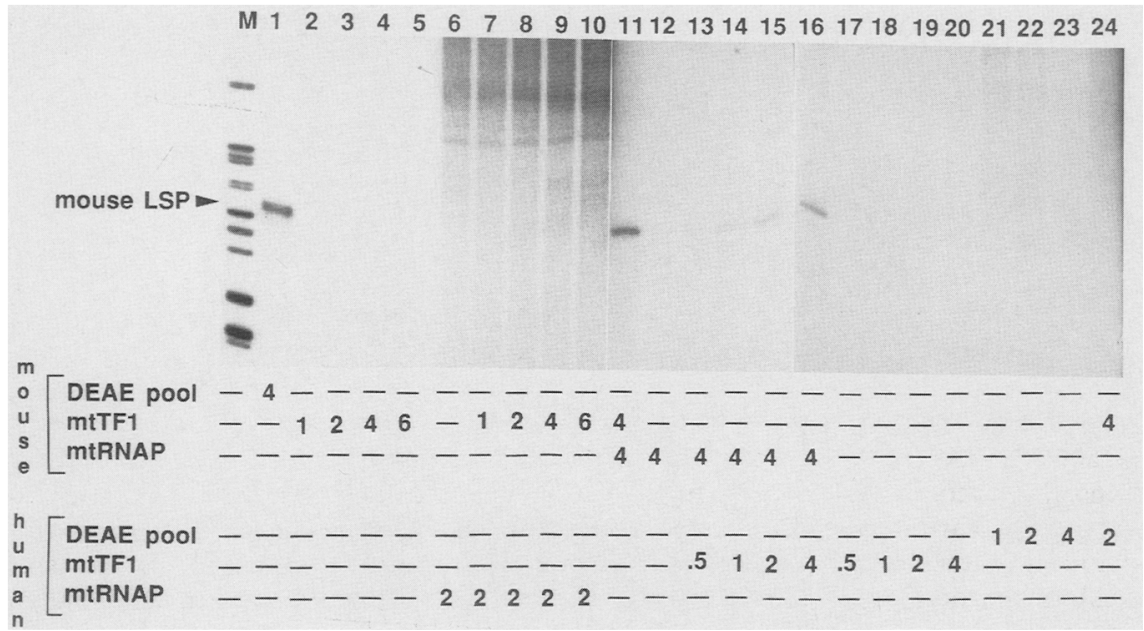

Figure 9. Heterologous in vitro transcription of the mouse mtDNA LSP. The mouse mtDNA deletion clone $\Delta 3^{\prime}-297$ (Chang and Clayton 1986b|, containing an intact LSP, was used to program transcription by various combinations of mouse and human mitochondrial protein fractions, as indicated below each lane. The fractions used were the same as in the experiment described in the legend to Fig. 7, with the addition of the human DEAE pool, equivalent to the mouse DEAE pool, which contains the bulk of both mtTF 1 and mtRNA polymerase activities. Complete runoff transcripts initiated correctly at the mouse LSP are $\sim 211$ nucleotides in length (arrowhead). The experimental design may be summarized as follows: (Lane 1) Homologous transcription by mouse DEAE pool alone; (lanes 2-5) a titration of mouse mtTFl alone; (lane 6) human mtRNA polymerase alone; (lanes 7-10) a titration of mouse mtTF1 in the presence of human polymerase; (lane 11) homologous, reconstituted transcription by mouse mtTF1 and mouse mtRNA polymerase; (lane 12) mouse polymerase alone; (lanes 13-16) a titration of human mtTFl in the presence of mouse mtRNA polymerase; (lanes 17-20) human mtTFl titrated in the absence of any added polymerase; (lanes 21-23) a titration of human DEAE pool; (lane 24) human DEAE pool plus mouse mtTFl; (lane M) a ${ }^{32}$ P-labeled HpaII digest of pBR322. Lanes 1-10 are of shorter exposure for ease of comparison.

This is confirmation of the fact that a functional assay for binding determinants on the DNA can identify bases where mutations are tolerated.

An extreme example may be the alignment of human and mouse mtTF1-binding sites shown in Figure 10B. Of the 22 bases in each site, only 7 are identical between the two species, but of these, 6 are contacted. Thus, mtTF1 serves as a sensitive protein 'probe' for homology between these two highly divergent sequences. That these two sequences may be related, albeit distantly, in spite of their apparent dissimilarity, is strongly suggested by their functional homology, here demonstrated by heterologous transcription and DNase I footprinting.

Having implied that human and mouse mtTF1binding sites are related, we hasten to point out that this relationship is quite distant. Although a majority of the conserved bases are contacted, only a minority of contacted bases are conserved. That mtTF1 from either species can locate and occupy the binding site of the other implies considerable flexibility in the mechanism of recognition. Such flexibility was prefigured by the demonstration that the human factor could bind and activate both the HSP and LSP of human mtDNA, two sequences that are only partially homologous (Fisher et al. 1987). Flexible sequence specificity may also explain one of the more unusual properties of $\mathrm{mtTF} 1$, namely, its relatively high affinity for nonspecific DNA. We noted previously that mtTF1 lacked the stringency exhibited by most eukaryotic nuclear transcription factors (Fisher and Clayton 1988); the explanation we offered was that the mitochondrial milieu demanded less specificity of a transcriptional activator, because mtDNA is generally a homogeneous population of 16.5 - $\mathrm{kb}$ closed circular molecules.

Our present results render alternative explanations more likely. In one scenario, the rapid genomic evolution of mtDNA sequences, which is most accelerated in the displacement-loop region containing the transcriptional promoters (Foran et al. 1988), may select for flexibility in the proteins that must interact with these control elements. This flexibility may, in turn, serve a regulatory function - the differential expression of L-strand and $\mathrm{H}$-strand genes - that would otherwise require additional promoter-specific factors. (However, we have not ruled out the existence of such factors.) Because of the high mutation rate of its target sequence and the need to recognize multiple sites with different affinities, mtTF1 may have had neither evolutionary time nor sufficient selective pressure to develop tighter sequence specificity for the LSP or HSP sequence. Conversely, promoter divergence may be a consequence, rather than a cause, of mtTF1's flexibility; the factor may be relatively ineffective in selecting against mutations in its binding site. We cannot distinguish between these two evolutionary mechanisms; however, we suspect some relationship between promoter divergence and flexible recognition.

Sequence specificity that is both flexible and evolutionarily mutable could be achieved by maintaining a high affinity for nonspecific DNA and/or a degree of degeneracy in the specific binding site. At many sites in 

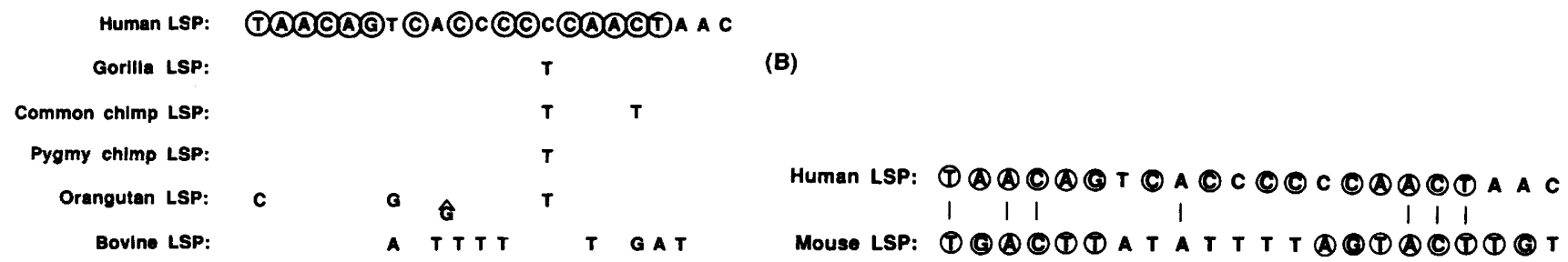

Figure 10. Evolution of the mitochondrial LSP. (A) Phylogenetic comparison of the human LSP mtTFl-binding site with putative LSP regions of four other ape mtDNAs (Foran et al. 1988; W.M. Brown, pers. comm.) and with a putative LSP of bovine mtDNA (King and Low 1987) reveals considerable conservation of bases contacted by human mtTF1 in its homologous target sequence (top). (Circles denote an mtTF1 contact on the coding strand or its complement on the noncoding strand.) On each line below the human sequence, only those positions that differ in each species are shown. In orangutan mtDNA, a one-base insertion has occurred (J.L. Boore and W.M. Brown, pers. comm.); this is indicated by the G drawn below the line showing substitutions. $(B)$ Direct comparison of contacts made by human and mouse mtTFls at their respective LSPs reveals previously undetected homologies between the two sequences. Only coding strand sequences are shown, but circled bases indicate both direct contacts with coding strand purine residues and contacts with complementary residues on the noncoding strands. Vertical lines are drawn between all bases conserved between mouse and human LSPs, whether contacted or not. Sequences are aligned as in Fig. 4.

the LSP, methylation causes only a moderate destabilization of mtTF1-LSP complexes (see Figs. 2 and 3). Presumably, tight binding is the cumulative result of multiple low affinity interactions with these bases. How mtTF1 may evolve to compensate for gradual mutation of its target sequence is also suggested by the three-dimensional maps of contacted bases on the DNA of the mouse and human LSPs presented in Figure 5. It is conceivable that the protein may adjust to target site mutation by discrete point mutations of amino acids within a conserved matrix, i.e., a stable (with respect to secondary structure) DNA-binding domain. Indeed, there may be precedents for such a model. Guarente and coworkers have established that a yeast transcriptional activator, HAP1, uses the same DNA-binding domain to recognize two divergent DNA sequences (Pfeifer et al. 1989|. Furthermore, the octamer-binding transcription factors are examples of proteins that can interact with different, but related, recognition sites (see GarcíaBlanco et al. 1989). An intriguing possibility is that flexibility is built into mtTF1's DNA-binding domain(s) and is reflected in its contact array. Major groove recognition that depends solely on the N7 position of purine residues would be naturally insensitive to transitions, whereas binding to $\mathrm{A} \rightarrow \mathrm{T}$ base pairs in the minor groove might tolerate an $\mathrm{A} \rightarrow \mathrm{T}$ to $\mathrm{T} \rightarrow \mathrm{A}$ transversion, as described by Seeman et al. (1976). Recognition based on these two features alone could accommodate the brisk mutation rate of mtDNA with little or no change in the amino acid sequence of the protein. However, a rigorous test of this model would require a specific assay for every potential site of base pair discrimination in both the major and minor grooves.

\section{Mitochondrial RNA polymerase may be species specific}

What, then, accounts for the stringent species specificity of mitochondrial transcription? The DNA-binding tran- scription factor $\mathrm{mtTF} 1$ is able to recognize and activate a heterologous LSP (Figs. 6-9), and there apparently is no major barrier to protein-protein interactions across species lines; human mtRNA polymerase can be stimulated by mouse mtTF1, and human mtTFl can activate transcription by mouse mtRNA polymerase, albeit at lower efficiencies in the heterologous cases. Incompatibility may therefore be at the level of protein-DNA recognition. Having found that mtTFl does not confer species specificity, we must conclude that selectivity resides in some other component of the transcriptional apparatus. Moreover, we can narrow our search for the species-specificity factor to the chromatographic fraction containing the mtRNA polymerase. That the polymerase itself might determine specificity is an intriguing possibility with both experimental support and precedent. In human mitochondrial extracts depleted of mtTF1, a very low level of accurate initiation is observed that is independent of upstream sequences recognized by $\mathrm{mtTF} 1$; the promoter domain encompassing the start site is absolutely required, however, as it is for mtTF1-dependent transcription even when the upstream mtTF1-binding site is intact (Fisher et al. 1987). We have previously proposed that this minimal 'core' promoter, which is not conserved between mouse and human mtDNA, might represent a polymerase recognition element. Furthermore, the mtRNA polymerase of Saccharomyces cerevisiae is homologous to the RNA polymerases encoded by phages T7, T3, and SP6 (Masters et al. 1987). These closely related polymerases are remarkable for their ability to recognize their respective promoters faithfully in the absence of accessory factors; however, despite the extensive homology among the three proteins, none is able to recognize another's cognate promoter efficiently (Dunn et al. 1971; McGraw et al. 1985). Thus, these phage polymerases may continue to provide a useful paradigm for their eukaryotic relatives, the mtRNA polymerases. 
Species specificity of mammalian mtDNA transcription: contrasts and parallels with other systems

A current theme in the field of eukaryotic transcriptional regulation is the conservation of DNA control elements and trans-acting factors between distant species. Homology, both structural and functional, has been established between a yeast DNA-binding transcriptional activator, GCN4, and the product of the avian oncogene jun (Struhl 1987; Vogt et al. 1987), which is, in turn, a homolog of the human cellular transcription factor, AP-1 (Bohmann et al. 1987). Conservative evolution of transcriptional effector molecules is also suggested by the creation of a hybrid heteromeric protein containing subunits of the human CCAAT-binding protein CP1 and the yeast HAP2/HAP3 CCAAT-binding transcriptional activator that retains specificity for CCAAT elements (Chodosh et al. 1988). This picture derives primarily from analysis of transcription catalyzed by the nuclear RNA polymerase II (for review, see Guarente 1988) but may apply equally to RNA polymerase III (Emerson and Roeder 1984).

In contrast, transcription of eukaryotic rDNA by nucleolar RNA polymerase I shows a much higher degree of species specificity; mitochondrial transcription may be analogous to rDNA transcription in this respect. Indeed, the parallels between the two transcription systems are extensive. Both can utilize small promoters, in most cases encompassing the transcriptional start sites and 30-50 bp of upstream sequence; in addition, more distal upstream elements have been found in polymerase I promoters by using in vivo microinjection techniques not feasible for mtDNA and by sensitive template competition experiments not yet attempted for mitochondrial transcription (however, for evidence of distal upstream elements of mouse mtDNA promoters, see Chang and Clayton 1986b). Both mtRNA polymerase and RNA polymerase I synthesize polycistronic precursor RNAs that are processed to yield multiple mature forms (the mitochondrial $\mathrm{H}$-strand transcript actually encodes the two mitochondrial rRNAs). Both mtDNA and rDNA are present in high copy number in eukaryotic cells, and both are localized within specialized organelles. Finally, there is considerable similarity between the two systems at the molecular level of protein-DNA recognition. In their studies of the rDNA transcription of Acanthamoeba, Paule and co-workers have described sequence-specific protein-DNA interactions that resemble those seen in human and mouse mtDNA transcription. A transcription initiation factor (TIF) binds to the rDNA promoter between 12 and $69 \mathrm{bp}$ upstream of the start site. RNA polymerase then binds adjacent to the TIF, occluding sequences that encompass the transcriptional start site by a non-sequence-specific mechanism (perhaps through interaction with TIF). When provided with rNTPs, polymerase I initiates transcription and vacates the promoter region; initiation, unlike polymerase binding, requires specific DNA sequences at the start site (Kownin et al. 1987). The gross anatomy of the human and mouse mitochondrial promoters is very similar, as described above and in previous reports /Chang and Clayton 1986b; Fisher et al. 1987; Topper and Clayton 1989). Upstream control regions are positioned similarly in mtDNA and rDNA; a requirement for specific nucleotides at the transcriptional start site has been documented for three of the four mammalian mitochondrial promoters characterized (the mouse HSP is an exception; Chang and Clayton 1986c), although stable binding of mtRNA polymerase to promoter DNA or to a mtTF1-DNA complex has not been observed. There may be similarities at the fine structural level as well. The mtTF1-binding site of the human LSP can be dissected into two domains by linker substitution analysis; these domains correlate well with the two directly repeated 5'-PyAAC-3' elements contacted by mtTF1 near the boundaries of the control region. Disruption of the sequence between the two repeats had a somewhat less pronounced effect on both mtTF1 binding and transcription; the contacted bases mutated by this linker substitution fall primarily within the cluster of six consecutive $G$ residues between -31 and -26 (Fisher et al. 1987; Topper and Clayton 1989). In addition, contacts between mouse mtTFl and the LSP seem to fall in two separate domains (Fig. 3). In their linker scan of the Xenopus rDNA promoter, Windle and Sollner-Webb (1986) found evidence for distinct domains-similarly situated-of the polymerase I core promoter; two regions essential for promoter recognition were separated by a short stretch where mutations were better tolerated.

If species specificity of mitochondrial transcription depends on the polymerase, the analogy between mtDNA and rDNA transcription becomes less compelling. Tjian and co-workers have identified a dissociable factor, SL1, that can reprogram the heterologous polymerase I transcription machinery to recognize the promoter of the species, either mouse or human, from which the SLl originates (Learned et al. 1985). The mechanism appears to involve protein-protein interaction, leading to an alteration in the DNA-binding function of another factor (Learned et al. 1986). More recently, Safrany et al. (1989) have shown that determinants of transcriptional species specificity are located between positions -32 and -14 of the mouse rDNA promoter. A chimeric template, containing this mouse sequence substituted for its human counterpart in an otherwise human polymerase I promoter, was transcribed in a mouse extract; moreover, it was capable of binding a mouse polymerase I transcription factor, TFID. In mitochondria, the requirement for a homologous polymerase fraction could reflect the currently undetected presence of such a species-specific factor in our preparations; alternatively, this function may be incorporated into the polymerase itself in a more economical transcription system.

An exception to the rule of species-specific rDNA transcription has been reported; mouse and Xenopus rDNA promoters are both homologous in sequence and functionally interchangeable in vitro /Culotta et al. 
1987). It may be significant that mouse and Xenopus mtDNA HSPs also show significant homology (Bogenhagen and Romanelli 1988), although heterologous transcription in vitro has not been attempted. The amphibian mitochondrial promoters contain a dyad symmetry element not present in human mitochondrial promoters (Bogenhagen and Yoza 1986); in the murine LSP, such a dyad overlaps the mtTFl-binding site (Chang and Clayton 1986b) but is virtually untouched by the bound protein. The well-established association between inverted repeat motifs and protein binding makes this element a likely candidate to be a functional promoter domain, perhaps an RNA polymerase-binding or recognition site; its absence from the human LSP could point to a role in the evolution of species specificity. We must now turn our attention to these putative promoter elements and to studies of mtRNA polymerase, if we are to understand the remarkable evolutionary lability of mitochondrial transcription signals.

\section{Materials and methods}

\section{Construction of mtDNA templates for in vitro assays}

The plasmid constructs containing human mtDNA that were used for runoff transcription and DNase footprinting experiments have been described previously. The plasmid pKB741SP (Fisher and Clayton 1985) was digested with EcoRI to produce a template with an expected transcription product of 416 nucleotides initiated at the LSP. For DNase I footprint analysis and methylation interference studies, we used deletion clone L5' $\Delta-56$ (Chang and Clayton 1984), which contains an intact human LSP as the sole promoter on an $\sim 460$-bp mtDNA insert. This plasmid was linearized with restriction enzyme BamHI at the site of the deletion end point, $56 \mathrm{bp}$ upstream of the LSP start site, and end-labeled either with $\left[\gamma^{-32} \mathrm{P}\right] \mathrm{ATP}$ (New England Nuclear, Boston, Massachusetts) and T4 polynucleotide kinase (Pharmacia, Piscataway, New Jersey) or with $\left[\alpha^{-32}\right.$ P]dATP (New England Nuclear) and the large fragment of Escherichia coli DNA polymerase I (Pharmacia). An 140-bp end-labeled fragment containing the LSP was then isolated by Ball digestion and electrophoresis in, followed by extraction from, nondenaturing polyacrylamide gels, essentially as described previously (Fisher et al. 1987).

Mouse mtDNA constructs were derived from a series of BAL-31 deletions of parental plasmid pMHX 406 containing 406 bp of mouse mtDNA; the deleted fragments were subsequently inserted in vector pSP65 with EcoRI and HindIII linkers, as described previously (Chang and Clayton 1986b). When digested with HindIII and SphI, deletion clone $\Delta 3^{\prime}-297$ yielded an 827-bp fragment that supported production of an $\sim 210$-nucleotide Lstrand transcript. An additional transcription template, $\Delta 5^{\prime}-50$, containing only 50 bp upstream of the LSP, was utilized for several heterologous experiments with human mtTFl. The deletion clone $\Delta 5^{\prime}-88$ was digested with EcoRI, end-labeled, and cleaved either with HindIII to yield an $\sim 300$-bp fragment used in DNase I footprint analysis (Fig. 1) and runoff transcriptions (Fig. 10) or with $S s p I$ to generate an $\sim 125$-bp probe that was used both for methylation interference and heterologous DNase I footprint analysis.

\section{Fractionation of human mitochondrial transcription extracts}

Human mtTFl and mtRNA polymerase were isolated from human $\mathrm{KB}$ cell mitochondria, as described previously (Walberg and Clayton 1983; Fisher and Clayton 1988). Briefly, we per- formed sequential ion-exchange chromatography on a cleared mitochondrial lysate using DEAE-Sephacel (Pharmacia) and phosphocellulose P11 (Whatman, Clifton, New Jersey) resins. In addition, mtTFl was further purified by Mono $Q$ fast performance liquid chromatography (FPLC; Pharmacia).

\section{Fractionation of mouse mitochondrial transcription extracts}

$D E A E$ chromatography Mouse mitochondria were prepared from 30 liters of exponentially growing LA9 cells by the sucrose step gradient procedure (Bogenhagen and Clayton 1974). Isolation of mouse transcriptional activity was accomplished as described previously (Chang and Clayton 1986a), with several modifications. First, the mitochondria were lysed in $0.55 \%$ Triton X-100 in the presence of $0.6 \mathrm{M} \mathrm{KCl}$. The lysate was centrifuged for $1 \mathrm{hr}$ at $45,000 \mathrm{rpm}\left(130,000 \mathrm{~g}_{\mathrm{av}}\right)$ in a Ti75 rotor (Beckman Instruments, Palo Alto, California), and the supernatant (S-130) was collected and diluted to $0.1 \mathrm{M} \mathrm{KCl}$. The sample was applied to a 40-ml column of DEAE-Sephacel (Pharmacia) that was then washed with $0.1 \mathrm{M} \mathrm{KCl}$ buffer containing $10 \mathrm{mM}$ Tris- $\mathrm{Cl}$ (pH 8.0), $0.2 \mathrm{~mm}$ EDTA, 7.5\% glycerol, and $1.0 \mathrm{~mm}$ dithiothreitol (DTT). The bound proteins were eluted with $0.3 \mathrm{M}$ $\mathrm{KCl}$, and the collected fractions were assayed for protein concentration using the method of Schaffner and Weissman (1973) and for nonspecific RNA polymerase activity using a poly[d(AT)] template, as described previously (Fisher and Clayton 1985). Fractions containing transcriptional activity were pooled, and 2 $\mathrm{ml}$ of this pool (total volume, $30 \mathrm{ml}$ ) was dialyzed against 20 $\mathrm{mm}$ Tris- $\mathrm{Cl}(\mathrm{pH} 8.0), 50 \mathrm{~mm} \mathrm{KCl}, 1 \mathrm{~mm}$ DTT, $0.5 \mathrm{~mm}$ EDTA, and $50 \%$ glycerol and stored at $-20^{\circ} \mathrm{C}$. This dialysate is the DEAE pool fraction used in runoff transcription assays.

Phosphocellulose chromatography The remainder of the DEAE pool was loaded on a $1.5 \times 20$-cm column of phosphocellulose PIl (Whatman). After extensive washing with buffer containing $0.3 \mathrm{M} \mathrm{KCl}$, the bound proteins were eluted with a linear $0.3-1.0 \mathrm{M} \mathrm{KCl}$ gradient. The collected fractions were assayed for nonspecific transcription on a poly[d(A-T)] template and for the ability to form mtTF1-DNA complexes, as assayed by gel retardation (Fisher and Clayton 1988). The peak of mouse mtRNA polymerase activity eluted between 0.45 and $0.5 \mathrm{M}$ $\mathrm{KCl}$, whereas mouse mtTFl eluted between 0.7 and $0.8 \mathrm{M} \mathrm{KCl}$. Two pools were made, of fractions containing the bulk of polymerase and of mtTF1, respectively, and dialyzed, as described above.

\section{DNase I footprint analysis}

The DNase I protection assay described by Galas and Schmitz (1978) and modified by Fisher et al. (1987) was used to assess the sequence-specific binding of human or mouse mtTF1 to both $5^{\prime}$ - and $3^{\prime}$-end-labeled fragments of either the mouse LSP (clone $\Delta 5^{\prime}-88$; Chang and Clayton 1986b) or the human LSP. The only additional modification was the use of poly[d(I-C)] (Pharmacia) as a nonspecific carrier at a final concentration of 2 $\mu \mathrm{g} / \mathrm{ml}$ in each reaction. In heterologous experiments, mouse end-labeled LSP fragments were incubated with several concentrations of human mtTFl, as well as with a control sample of mouse mtTF1 prior to limited DNase I digestion and subsequent extraction, electrophoresis, and visualization by autoradiography. Analogous experiments were carried out on end-labeled human LSP fragments using mouse mtTF1.

\section{In vitro transcription assays}

In vitro transcription assays were carried out as described previously (Fisher et al. 1987). Specifically, the experiments were conducted with phosphocellulose-purified and dialyzed pools of 
human and mouse mtTF1 and mtRNA polymerase; the dialyzed DEAE pool also served as a source of polymerase in some experiments. In addition, some assays were performed with Mono Q-purified human mtTF1 fractions. The DNA concentration was $1 \mu \mathrm{g} / \mathrm{ml}$ in all experiments.

\section{Methylation interference analysis of protein-DNA contacts}

Methylation interference analyses were performed according to Hendrickson and Schleif (1985), with some modifications. For the methylation reactions, $5 \mu$ l of each labeled DNA probe $\left(\sim 1.5 \times 10^{6}\right.$ Cerenkov cpm) was added to $200 \mu \mathrm{l}$ of DMS buffer $150 \mathrm{~mm}$ sodium cacodylate $\left(\mathrm{pH} 8.0\right.$, $10 \mathrm{~mm} \mathrm{MgCl}_{2}, 1 \mathrm{~mm}$ EDTA], containing $1 \mu \mathrm{g}$ of E. coli tRNA (Boehringer-Mannheim) as carrier, and chilled to $0^{\circ} \mathrm{C}$. DMS (Eastman Kodak) was added at $1 \mu \mathrm{l}$ per reaction prior to a 7 -min incubation at $20^{\circ} \mathrm{C}$. The reactions were stopped with $50 \mu$ l DMS stop buffer [1.5 M sodium acetate $(\mathrm{pH} 7.0), 1.0 \mathrm{M} \beta$-mercaptoethanol], and the DNA was recovered by repeated precipitation with ethanol. Under these conditions, fewer than one site per molecule, on average, was methylated at either the $\mathrm{N} 7$ position of a guanine in the major groove or the N3 position of an adenine in the minor groove. DNA sequence ladders were produced by standard chemical sequencing reactions $(A+G, T+C$; Maxam and Gilbert 1977), using the same labeled fragments.

The methylated fragments containing either human or mouse LSP sequences were incubated with phosphocellulosepurified mtTFl from human or mouse mitochondria, respectively. The final DNA concentration was $\sim 0.1 \mu \mathrm{g} / \mathrm{ml}$, with $\sim 5 \times 10^{5}$ Cerenkov cpm present in each $80-\mu l$ reaction. The final concentration of $E$. coli tRNA was $\sim 2 \mu \mathrm{g} / \mathrm{ml}$. The amounts of transcription factor added were chosen to maximize one-to-one binding of mtTF1 to DNA, as determined by titration experiments (not shown), and are reported in the legends to Figures 2 and 3. After a 20 -min incubation at $28^{\circ} \mathrm{C}, 1.6 \mu \mathrm{l}$ of a $1-\mathrm{mg} / \mathrm{ml}$ solution of unlabeled plasmid DNA containing an intact homologous LSP (human, pKB741SP; mouse, $\Delta 3^{\prime}$-297) was added, resulting in a 200 -fold mass excess $(\sim 10$-fold molar excess) of the competitor relative to the labeled fragment. The samples were incubated for an additional $2 \mathrm{~min}$ at room temperature to allow dissociation of $\mathrm{mtTF} 1$ from nonspecific sites on the labeled fragment (Fisher and Clayton 1988), mixed with one-tenth volume of loading dye $150 \%$ glycerol, $0.1 \%$ bromphenol blue, $0.1 \%$ xylene cyanol), and loaded on a $4 \%$ polyacrylamide $(0.11 \%$ bis-acrylamide) nondenaturing gel that was running at $30 \mathrm{~mA}$. All reagents and conditions for gel electrophoresis of protein-DNA complexes were as described previously. Following electrophoresis, the wet gel was autoradiographed and slices containing bound and unbound fragment were excised. The DNA was electrophoresed onto a DEAE membrane (NA45, Schleicher \& Schuell), essentially as described by Baldwin and Sharp (1987), and eluted from the membrane by incubating in $400 \mu \mathrm{l}$ of $10 \mathrm{~mm}$ Tris- $\mathrm{Cl}(\mathrm{pH} 8.0), 1 \mathrm{M}$ $\mathrm{NaCl}$, and $1 \mathrm{~mm}$ EDTA at $65^{\circ} \mathrm{C}$ for $30 \mathrm{~min}$, followed by a rinse with $400 \mu \mathrm{l}$ of the same solution. Insoluble material was removed by centrifugation ( $30 \mathrm{~min}$ at $10,000 \mathrm{rpm}$ at $\left.4^{\circ} \mathrm{C}\right), 16 \mu \mathrm{g}$ of $E$. coli tRNA was added to the supernatant as carrier, and nucleic acids were precipitated with ethanol. Fragments were recovered by centrifugation, rinsed with $70 \%$ ethanol, and dried. Radioactivity recovered from each electrophoretic species was quantitated by Cerenkov counting, and samples were reacted with piperidine following pretreatment in $20 \mathrm{mM}$ ammonium acetate, $0.1 \mathrm{mM}$ EDTA, to cleave the DNA at $\mathrm{G}$ and A residues (G > A), as in sequencing (Maxam and Gilbert 1977). An aliquot of each methylated probe was ethanol-precipitated, Cerenkov-counted and subjected to G > A strand scission, without exposure to mtTFl or gel electrophoresis, to generate the markers electrophoresed in lanes G > A of Figures 2 and 3 . After repeated lyophilizations, the DNA was resuspended in $80 \%$ formamide, TBE [45 $\mathrm{mm}$ Tris-borate $\{\mathrm{pH} 8.3$ ), l $\mathrm{mm}$ EDTA], $0.1 \%$ bromphenol blue, and $0.1 \%$ xylene cyanol to give solutions of equal specific activity in Cerenkov cpm per microliter. Samples were denatured for $5 \mathrm{~min}$ at $70^{\circ} \mathrm{C}$ and electrophoresed in $6 \%$ or $10 \%$ polyacrylamide sequencing gels containing $7 \mathrm{M}$ urea in TBE. The gels were dried and used to expose Kodak XAR-5 X-ray film in the presence of Cronex intensifying screens (Dupont, Wilmington, Delaware).

\section{Computer graphics}

Three-dimensional graphics displays of mouse and human mtTF1 DNA contacts on their homologous promoters were based on methylation interference results. Uniform B-DNA structures (Arnott and Hukins 1972) for the LSP sequences were generated using the ENCAD package (M. Levitt, pers. comm.). The van der Waals radii of contacted $\mathrm{N} 7$ atoms of guanines and N3 atoms of adenines were displayed using the MOGLI molecular graphics software program projected on an Evans and Sutherland Picture System 300.

\section{Acknowledgments}

This investigation was supported by grant GM33088-19 from the National Institute of General Medical Sciences and grant NP-9O from the American Cancer Society, Inc. R.P.F. and M.A.P. are Medical Scientist Training Program Trainees of the National Institute of General Medical Sciences (GM07365-13). We acknowledge the contributions of Michael W. Gray, of Dalhousie University, Halifax, Nova Scotia, in the development of a mouse mtTFl isolation protocol while on sabbatical leave in this laboratory and of David D. Chang, formerly of this laboratory, for the gift of human and mouse mtDNA clones. We thank Miriam Hirshberg, Department of Cell Biology, for generating the B-DNA coordinates of LSP sequences and William $\mathrm{N}$. Hurja, manager of computer services in the Department of Cell Biology, for his generous instruction in the use of the computer graphics system and software. We also thank J.F. Hess and G.A. Breen for helpful comments on the manuscript.

\section{References}

Anderson, S., A.T. Bankier, B.G. Barrell, M.H.L. de Bruijn, A.R. Coulson, J. Drouin, I.C. Eperon, D.P. Nierlich, B.A. Roe, F. Sanger, P.H. Schreier, A.J.H. Smith, R. Staden, and I.G. Young. 1981. Sequence and organization of the human mitochondrial genome. Nature 290: 457-465.

Arnott, S. and D.W.L. Hukins. 1972. Optimised parameters for A-DNA and B-DNA. Biochem. Biophys. Res. Commun. 47: 1504-1509.

Baldwin, A.S., Jr. and P.A. Sharp. 1987. Binding of a nuclear factor to a regulatory sequence in the promoter of the mouse $\mathrm{H}-2 \mathrm{~K}^{\mathrm{b}}$ class I major histocompatibility gene. Mol. Cell. Biol. 7: 305-313.

Bibb, M.J., R.A. Van Etten, C.T. Wright, M.W. Walberg, and D.A. Clayton. 1981. Sequence and gene organization of mouse mitochondrial DNA. Cell 26: 167-180.

Bogenhagen, D. and D.A. Clayton. 1974. The number of mitochondrial deoxyribonucleic acid genomes in mouse L- and human HeLa cells. Quantitative isolation of mitochondrial deoxyribonucleic acid. J. Biol. Chem. 249: 7991-7995.

Bogenhagen, D.F. and M.F. Romanelli. 1988. Template sequences required for transcription of Xenopus laevis mitochondrial DNA from two bidirectional promoters. Mol. Cell. Biol. 8: 2917-2924.

Bogenhagen, D.F. and B.K. Yoza. 1986. Accurate in vitro tran- 
scription of Xenopus laevis mitochondrial DNA from two bidirectional promoters. Mol. Cell. Biol. 6: 2543-2550.

Bohmann, D., T.J. Bos, A. Admon, T. Nishimura, P.K. Vogt, and R. Tjian. 1987. Human proto-oncogene c-jun encodes a DNA binding protein with structural and functional properties of transcription factor AP-1. Science 238: 1386-1392.

Chang, D.D. and D.A. Clayton. 1984. Precise identification of individual promoters for transcription of each strand of human mitochondrial DNA. Cell 36: 635-643.

- 1986a. Identification of primary transcriptional start sites of mouse mitochondrial DNA: Accurate in vitro initiation of both heavy- and light-strand transcripts. Mol. Cell. Biol. 6: 1446-1453.

1986b. Precise assignment of the light-strand promoter of mouse mitochondrial DNA: A functional promoter consists of multiple upstream domains. Mol. Cell. Biol. 6: $3253-3261$.

. 1986c. Precise assignment of the heavy-strand promoter of mouse mitochondrial DNA: Cognate start sites are not required for transcriptional initiation. Mol. Cell. Biol. 6: $3262-3267$.

Chang, D.D., T.W. Wong, J.E. Hixson, and D.A. Clayton. 1985. Regulatory sequences for mammalian mitochondrial transcription and replication. In Achievements and perspectives of mitochondrial research: Biogenesis (ed. E. Quagliariello, E.C. Slater, F. Palmieri, C. Saccone, and A.M. Kroon), vol. 2, pp. 135-144. Elsevier Science Publishers, Amsterdam.

Chodosh, L.A., J. Olesen, S. Hahn, A.S. Baldwin, L. Guarente, and P.A. Sharp. 1988. A yeast and a human CCAAT-binding protein have heterologous subunits that are functionally interchangeable. Cell 53: 25-35.

Clayton, D.A. 1984. Transcription of the mammalian mitochondrial genome. Annu. Rev. Biochem. 53: 573-594.

Culotta, V.C., V.K. Wilkinson, and B. Sollner-Webb. 1987. Mouse and frog violate the paradigm of species-specific transcription of ribosomal RNA genes. Proc. Natl. Acad. Sci. 84: 7498-7502.

Dunn, J.J., F.A. Bautz, and E.K. Bautz. 1971. Different template specificities of phage T3 and T7 RNA polymerases. Nat. New Biol. 230: 94-96.

Emerson, B.M. and R.G. Roeder. 1984. DNA sequences and transcription factor interactions of active and inactive forms of mammalian 5S RNA genes. $J$. Biol. Chem. 259: 79267935.

Fisher, R.P. and D.A. Clayton. 1985. A transcription factor required for promoter recognition by human mitochondrial RNA polymerase. Accurate initiation at the heavy- and light-strand promoters dissected and reconstituted in vitro. J. Biol. Chem. 260: 11330-11338.

- 1988. Purification and characterization of human mitochondrial transcription factor 1. Mol. Cell. Biol. 8: 34963509.

Fisher, R.P., J.N. Topper, and D.A. Clayton. 1987. Promoter selection in human mitochondria involves binding of a transcription factor to orientation-independent upstream regulatory elements. Cell 50: 247-258.

Foran, D.R., J.E. Hixson, and W.M. Brown. 1988. Comparisons of ape and human sequences that regulate mitochondrial DNA transcription and D-loop DNA synthesis. Nucleic Acids Res. 16: 5841-5861.

Galas, D.J. and A. Schmitz. 1978. DNase footprinting: A simple method for the detection of protein-DNA binding specificity. Nucleic Acids Res. 5: 3157-3170.

García-Blanco, M.A., R.G. Clerc, and P.A. Sharp. 1989. The DNA-binding homeo domain of the Oct-2 protein. Genes Dev. 3: 739-745.
Guarente, L. 1988. UASs and enhancers: Common mechanism of transcriptional activation in yeast and mammals. Cell 52: 303-305.

Hendrickson, W. and R. Schleif. 1985. A dimer of AraC protein contacts three adjacent major groove regions of the araI DNA site. Proc. Natl. Acad. Sci. 82: 3129-3133.

King, T.C. and R.L. Low. 1987. Mapping of control elements in the displacement loop region of bovine mitochondrial DNA. J. Biol. Chem. 262: 6204-6213.

Kownin, P., E. Bateman, and M.R. Paule. 1987. Eukaryotic RNA polymerase I promoter binding is directed by protein contacts with transcription initiation factor and is DNA sequence-independent. Cell 50: 693-699.

Learned, R.M., S. Cordes, and R. Tjian. 1985. Purification and characterization of a transcription factor that confers promoter specificity to human RNA polymerase I. Mol. Cell. Biol. 5: 1358-1369.

Learned, R.M., T.K. Learned, M.M. Haltiner, and R.T. Tjian. 1986. Human rDNA transcription is modulated by the coordinate binding of two factors to an upstream control element. Cell 45: 847-857.

Masters, B.S., L.L. Stohl, and D.A. Clayton. 1987. Yeast mitochondrial RNA polymerase is homologous to those encoded by bacteriophages T3 and T7. Cell 51: 89-99.

Maxam, A.M. and W. Gilbert. 1977. A new method for sequencing DNA. Proc. Natl. Acad. Sci. 74: 560-564.

McGraw, N.J., J.N. Bailey, G.R. Cleaves, D.R. Dembinski, C.R. Gocke, L.K. Joliffe, R.S. MacWright, and W.T. McAllister. 1985. Sequence and analysis of the gene for bacteriophage T3 RNA polymerase. Nucleic Acids Res. 13: 6753-6766.

Pfeifer, K., K.-S. Kim, S. Kogan, and L. Guarente. 1989. Functional dissection and sequence of yeast HAP1 activator. Cell 56: $291-301$.

Safrany, G., N. Tanaka, T. Kishimoto, Y. Ishikawa, H. Kato, R. Kominami, and M. Muramatsu. 1989. Structural determinant of the species-specific transcription of the mouse rRNA gene promoter. Mol. Cell. Biol. 9: 349-353.

Schaffner, W. and C. Weissman. 1973. A rapid, sensitive, and specific method for the determination of protein in dilute solution. Anal. Biochem. 56: 502-514.

Seeman, N.C., J.M. Rosenberg, and A. Rich. 1976. Sequencespecific recognition of double helical nucleic acids by proteins. Proc. Natl. Acad. Sci. 73: 804-808.

Siebenlist, U. and W. Gilbert. 1980. Contacts between Escherichia coli RNA polymerase and an early promoter of phage T7. Proc. Natl. Acad. Sci. 77: 122-126.

Struhl, K. 1987. The DNA-binding domains of the jun oncoprotein and the yeast GCN4 transcriptional activator protein are functionally homologous. Cell 50: 841-846.

Topper, J.N. and D.A. Clayton. 1989. Identification of transcriptional regulatory elements in human mitochondrial DNA by linker substitution analysis. Mol. Cell. Biol. 9: $1200-1211$.

Vogt, P.K., T.J. Bos, and R.F. Doolittle. 1987. Homology between the DNA-binding domain of the GCN4 regulatory protein of yeast and the carboxyl-terminal region of a protein coded for by the oncogene jun. Proc. Natl. Acad. Sci. 84: 3316-3319.

Walberg, M.W. and D.A. Clayton. 1983. In vitro transcription of human mitochondrial DNA. Identification of specific lightstrand transcripts from the displacement-loop region. J. Biol. Chem. 258: 1268-1275.

Windle, J.J. and B. Sollner-Webb. 1986. Two distant and precisely positioned domains promote transcription of Xenopus laevis rRNA genes: Analysis with linker-scanner mutants. Mol. Cell. Biol. 6: 4585-4593. 


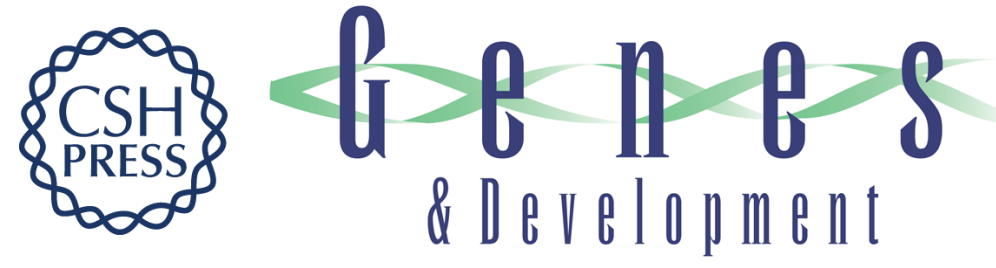

\section{Flexible recognition of rapidly evolving promoter sequences by mitochondrial transcription factor 1.}

R P Fisher, M A Parisi and D A Clayton

Genes Dev. 1989, 3:

Access the most recent version at doi:10.1101/gad.3.12b.2202

References This article cites 42 articles, 24 of which can be accessed free at:

http://genesdev.cshlp.org/content/3/12b/2202.full.html\#ref-list-1

License

Email Alerting

Service

Receive free email alerts when new articles cite this article - sign up in the box at the top right corner of the article or click here.

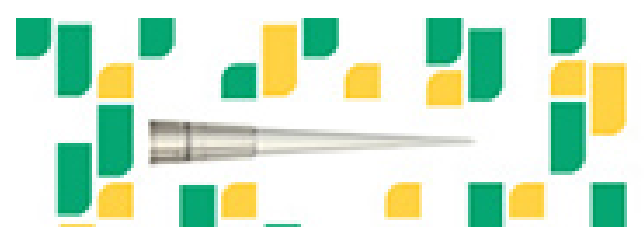

Focused on your science.

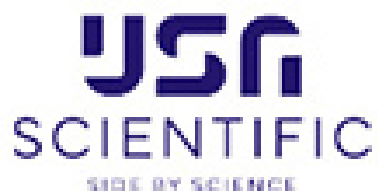

Copyright @ Cold Spring Harbor Laboratory Press 